\title{
Redundancy calibration of phased-array stations
}

\author{
P. Noorishad ${ }^{1,2}$, S. J. Wijnholds ${ }^{2}$, A. van Ardenne ${ }^{2,3}$, and J. M. van der Hulst ${ }^{1}$ \\ 1 Kapteyn Astronomical Institute, University of Groningen, Landleven 12, 9747 AV Groningen, The Netherlands \\ e-mail: noorishad@astro.rug.nl \\ 2 ASTRON, Oude Hoogeveensedijk 4, 7991 PD Dwingeloo, The Netherlands \\ 3 Chalmers University of Technology, 41296 Gothenburg, Sweden
}

Received 21 February 2012 / Accepted 1 May 2012

\begin{abstract}
Aims. We assess the benefits and limitations of using the redundant visibility information in regular phased-array systems to improve the quality of the calibration.

Methods. Regular arrays offer the possibility of using redundant visibility information to constrain the calibration of the array independently of a sky model and a beam model of the station elements. This requires a regular arrangement of the configuration of array elements and identical beam patterns.

Results. We revised a previously developed calibration method for phased-array stations using the redundant visibility information in the system and applied it successfully to a LOFAR station. The performance and limitations of the method were demonstrated by comparing its application to real and simulated data. The main limitation is the mutual coupling between the station elements, which leads to non-identical beams and stronger baseline-dependent noise. Comparing the variance in the estimated complex gains with the Cramer-Rao Bound indicates that redundancy is a stable and optimum method for calibrating the complex gains of the system. Conclusions. Our study shows that the use of the redundant visibility does improve the quality of the calibration in phased-array systems. In addition, it provides a powerful tool obtaining system diagnostics. Our results demonstrate that designing redundancy in both the station layout and the array configuration of future aperture arrays is strongly recommended. This is particularly true in the case of the Square Kilometre Array (SKA) with its dynamic range requirement that surpasses any existing array by an order of magnitude.
\end{abstract}

Key words. instrumentation: interferometers - telescopes - methods: observational

\section{Introduction}

An important conceptual difference between the next generation of radio telescopes and conventional ones is their hierarchical system architecture. An excellent example is the Low Frequency ARray (LOFAR), de Vos et al. (2009). In LOFAR, a station consists of phased arrays that are either sparse or dense. In the sparse phased-array stations, the station elements are dipoles that are digitally beam-formed to synthesize a station as a dish such as the low band antennas (LBAs) in a LOFAR station. Phased arrays operating above $\sim 100 \mathrm{MHz}$ are often implemented as compound elements or tiles, such as the high band antennas (HBAs) at the LOFAR stations or in Electronic Multi-Beam Radio Astronomy ConcEpt (EMBRACE), Ardenne et al. (2004); Kant et al. (2011), the latter being is an example of a very dense phased-array station. In these stations, the station elements are phased-array tiles (e.g. Fig. 1). A tile is a regular arrangement of many dipoles whose signals are added in phase to form an instantaneous beam (the first level of beam-forming). The tile output signals are digitally phased up to synthesize a station as a dish. At the next level in the beam-forming hierarchy, the beamformed output of each station is transported to the central correlator to synthesize the whole telescope. Calibration has to be performed at different levels of this hierarchy to provide a final, high dynamic range image of part of sky, as explained by Wijnholds et al. (2010).

In this paper, we concentrate on the calibration at station level to ensure that the station beam is stable over time and

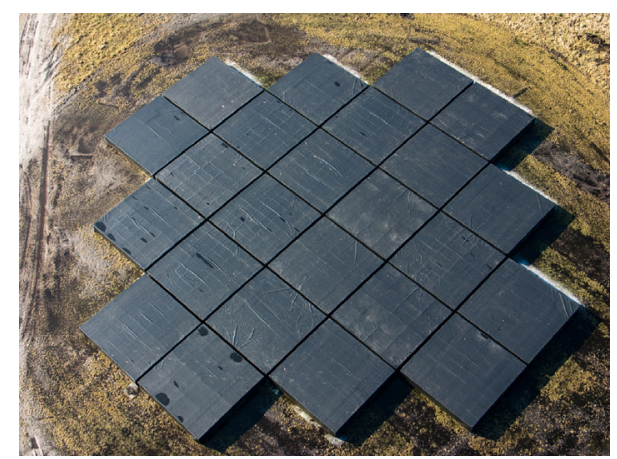

Fig. 1. Twenty-four-tile HBA station. Each tile is one station element. This is the station configuration for most of LOFAR's HBA stations, including the LOFAR core (CS302). It is clear that a station like this is highly redundant.

frequency. A robust calibration as part of the beam-forming process should guarantee a stable beam pattern of the station for data going to the central correlator. This is crucial for the dynamic range of the final images made using data from the entire array.

In a phased-array station, the output of all station elements can be correlated. These correlations are called station visibilities, which are used for engineering purposes e.g. station calibration and radio frequency interference (RFI) detection/mitigation. These correlations include many short baselines on which extended structures such as the Galactic plane are captured. 


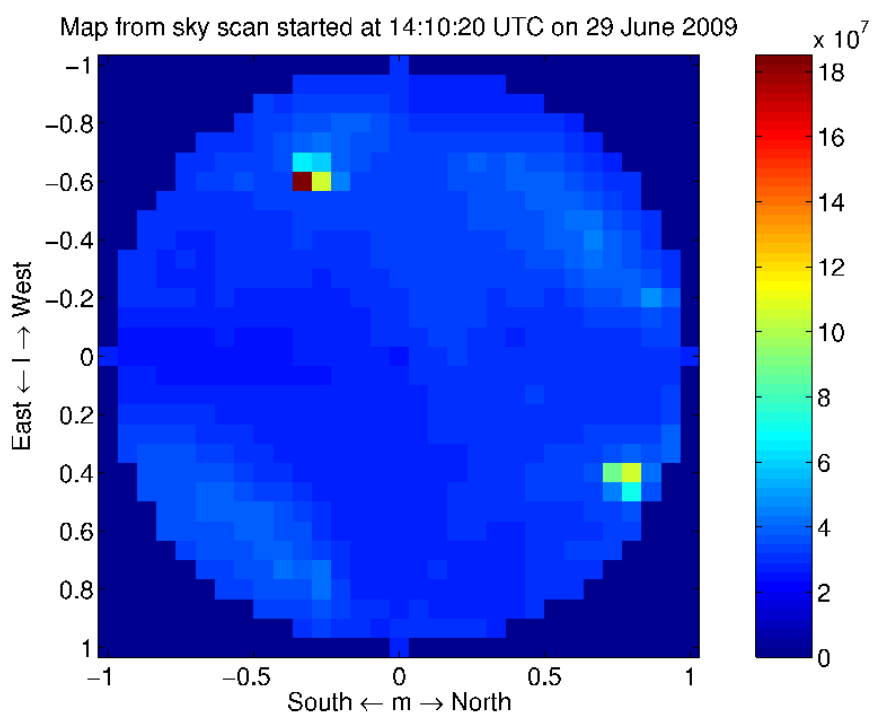

Fig. 2. The sky imaged by HBA tiles at 14:10:20 UTC on 29 June 2009. The Galactic plane appears in the north-west, the Sun appears in the south-west. One can also see their corresponding grating response in the image. The image is presented in the $(l, m)$-coordinates $l=$ $\cos (e l) \sin (a z)$ and $m=\cos (e l) \cos (a z)$, where $e l$ and $a z$ denote elevation and azimuth, respectively.

The most commonly used calibration methods for phased-array stations are model-based, such as a multi-source calibration method introduced by Wijnholds \& van der Veen (2009a). A model-based calibration method requires the presence of one or more relatively unresolved point sources such as CasA and a model of the extended structures (see Fig. 2). Modeling an extended structure is computationally difficult and expensive. Wijnholds \& van der Veen (2009b) phenomenologically model it as correlated noise and estimate the parameters of interest for calibration using a weighted alternative least squares (WALS) approach. However, the model-based methods are in general iterative methods, which usually converge after several iterations.

A regular arrangement of station elements has the advantage that it provides redundant baselines, i.e. baselines of the same physical length and orientation. The use of redundant baseline information for calibration was introduced by Noordam \& de Bruyn (1982). Its linearity, independence of a sky model, low computational cost, and proven efficiency for precision calibration of Westerbork Synthesis Radio Telescope (WSRT) observations motivated us to apply the redundancy calibration to phasedarray stations. The redundancy calibration algorithm uses the data of all redundant baselines to obtain a convergent calibration solution in a single step.

However, redundancy calibration in phased-array systems requires additional considerations. This is essentially because of their different design concepts e.g. the closely located elements of a phased-array station experience mutual coupling between elements that leads to non-identical beams of the station elements and to correlated receiver noise.

In this paper, we refine the standard data model presented in the phased-array signal processing literature to account for baseline-dependent corruptions in terms of the coupling effects. Using this refined data model, we briefly introduce the two calibration methods i.e. model-based and redundancy. This helps us to achieve a clearer understanding of the potential and limitations of both calibration methods. We also revise the redundancy method formalism presented by
(Wieringa 1991; Liu et al. 2010) to capture the nature of baseline dependent errors that affect the calibration accuracy. Some implementation issues are raised and investigated using observed and simulated data of LOFAR HBA stations. We evaluate the redundancy calibration performance by comparing the variance in its results with the Cramer-Rao Bound (CRB) and the plots of residuals for the corrected data after redundancy calibration. We also discuss limiting factors for its applicability.

Although we used HBA data to demonstrate the applicability and efficiency of the redundancy method, the analysis in this paper is relevant to any phased array that is to be calibrated using redundant visibility information.

We denote vectors in bold lowercase letters and matrices in bold uppercase letters. The matrix transpose and Hermitian transpose are denoted by $(.)^{\mathrm{T}}$ and $(.)^{\mathrm{H}}$, respectively. Operator $\operatorname{diag}($.$) creates a diagonal matrix of a given vector.$ Operator vec(.) creates a vector. Operator $\odot$ denotes elementwise matrix multiplication. Operator $\angle($.) returns the angle or phase of a complex number. Operator $\kappa($.$) returns the condition$ number of a matrix.

\section{Methods}

\subsection{Data model for phased arrays}

The standard data model for phased-array stations presented in the literature assumes that in the absence of RFI and any coupling effects, a phased array of $p$ elements has a signal vector, $\boldsymbol{x}(t)=\left[x_{1}(t), x_{2}(t), \ldots, x_{p}(t)\right]^{\mathrm{T}}$ that can be expressed as $\boldsymbol{x}(t)=\boldsymbol{\Gamma} \boldsymbol{\Phi}\left(\sum_{k=1}^{q} \boldsymbol{a}_{k} s_{k}(t)\right)+\boldsymbol{n}(t)=\boldsymbol{\Gamma} \boldsymbol{\Phi} \mathbf{A} \boldsymbol{s}(t)+\boldsymbol{n}(t)$,

where $s(t)$ is a $q \times 1$ vector containing $q$ mutually independent i.i.d. ${ }^{1}$. The Gaussian signals impinge on the array with the covariance of $\boldsymbol{\Sigma}_{\mathrm{s}}=\operatorname{diag}\left(\boldsymbol{\sigma}_{\mathrm{s}}\right)(\operatorname{size} q \times q)$, where $\sigma_{\mathrm{s}}$ is a vector of the source fluxes. They are also assumed to be narrow band, so we can define the $q$ spatial signature vectors $\boldsymbol{a}_{k}$, which include both the phase delays due to the geometry and the directional response of the receiving elements (assumed to be identical). The vectors $\boldsymbol{a}_{k}$ are called the array response vectors which, are usually normalized. The receiver noise signals $n_{i}(t)$ are assumed to be mutually independent i.i.d. Gaussian signals in a $p \times 1$ vector $\boldsymbol{n}(t)$ and uncorrelated. Thus, the noise covariance matrix, $\boldsymbol{\Sigma}_{n}=\operatorname{diag}\left(\boldsymbol{\sigma}_{n}\right)(\operatorname{size} p \times p)$. The amplitudes and the phases of direction-independent complex gains $\left(g_{i}\right.$ 's), that have to be calibrated are $\gamma=\left[\gamma_{1}, \gamma_{2}, \ldots, \gamma_{p}\right]^{\mathrm{T}}$ and $\boldsymbol{\phi}=\left[\mathrm{e}^{j \phi_{1}}, \mathrm{e}^{j \phi_{2}}, \ldots, \mathrm{e}^{j \phi_{p}}\right]^{\mathrm{T}}$. Correspondingly, $\boldsymbol{\Gamma}=\operatorname{diag}(\boldsymbol{\gamma})$ and $\boldsymbol{\Phi}=\operatorname{diag}(\boldsymbol{\phi})$. In addition, $\mathbf{A}=\left[\boldsymbol{a}_{1}, \boldsymbol{a}_{2}, \ldots, \boldsymbol{a}_{q}\right]($ size $p \times q)$ is a stack of the array response vectors. Before computing the coherency, $\boldsymbol{x}(t)$ is sampled with period $T$. The $n$th sample of the array signal vector $\boldsymbol{x}[n]$ is given by

$x[n]=\sum_{-\infty}^{\infty} x(t) \delta(t-n T)=x(n T)$,

$N$ samples can be stacked in a matrix $\mathbf{X}=[\boldsymbol{x}[1], \boldsymbol{x}[2], \ldots, \boldsymbol{x}[N]]$ (size $p \times N)$, which denotes the short-term integration data set or snapshot. The array covariance matrix or the visibility matrix describing the correlation between all sampled voltages can be estimated by $\hat{\mathbf{R}}=\mathbf{X} \mathbf{X}^{\mathrm{T}} / N$, whose expected value becomes either

$\mathbf{R}=\boldsymbol{\Gamma} \boldsymbol{\Phi} \mathbf{A} \boldsymbol{\Sigma}_{\mathrm{s}} \mathbf{A}^{\mathrm{H}} \boldsymbol{\Phi}^{\mathrm{H}} \boldsymbol{\Gamma}^{\mathrm{H}}+\boldsymbol{\Sigma}_{n}$

1 Temporally independent and identically distributed. 
or

$$
\mathbf{R}=\mathbf{G} \mathbf{A} \boldsymbol{\Sigma}_{\mathrm{s}} \mathbf{A}^{\mathrm{H}} \mathbf{G}^{\mathrm{H}}+\boldsymbol{\Sigma}_{n} .
$$

Correlator errors can be represented as an additive term in the covariance matrix, i.e. as a non-diagonal matrix. However, in Eq. (3) and throughout this paper, they are disregarded assuming that the correlator is designed perfectly.

We note that $\gamma_{i}$ could accommodate the overall amplitude gain of both the receiver system and the atmospheric disturbances, and $\phi_{i}$ the corresponding phase shift. In the case of station calibration, we do not calibrate for direction-dependent effects because we use snapshot data. In model-based methods, these are absorbed in the known sky. In the case of redundancy calibration, these are absorbed in its fundamental assumption. We elaborate on this assumption in Sect. 2.3.

In phased-arrays such as the HBA stations of LOFAR, or EMBRACE, the antenna elements are closely packed. This may cause mutual coupling between them, i.e. not all the power received by the elements is absorbed but some of the power is reradiated to the other elements. The reradiated power induces new currents in the other elements. Consequently, the radiation pattern of the elements changes. This leads to a lack of similarity between the element beam patterns or different array response vectors despite their physical identicalness (as considered in A). This effect can be modeled as a direction-dependent gain, $\mathbf{G}_{0}$ (size $p \times q$ ) being element-wise multiplied by the array response vectors

$$
\mathbf{R}=\mathbf{G}\left(\mathbf{G}_{0} \odot \mathbf{A}\right) \boldsymbol{\Sigma}_{\mathrm{s}}\left(\mathbf{G}_{0} \odot \mathbf{A}\right)^{\mathrm{H}} \mathbf{M}^{\mathrm{H}} \mathbf{G}^{\mathrm{H}}+\boldsymbol{\Sigma}_{n} .
$$

As studied by Svantesson (1998), mutual coupling can be directly represented by $\mathbf{M}($ size $p \times p)$ in the data model

$$
\mathbf{R}=\mathbf{G M A} \boldsymbol{\Sigma}_{\mathrm{s}} \mathbf{A}^{\mathrm{H}} \mathbf{M}^{\mathrm{H}} \mathbf{G}^{\mathrm{H}}+\boldsymbol{\Sigma}_{n} .
$$

One may note that a direct association of $\mathbf{M}$ and $\mathbf{G}_{0}$ cannot be expressed analytically. It requires a numerical evaluation that we present in Sect. 3.1. For simplicity and to continue our argument, we use the data model in Eq. (6).

Mutual coupling may not only act on the signal, but also on the system noise. The low noise amplifiers (LNAs) connected to the antennas in such an array, generate electromagnetic (EM) noise waves towards their outputs, but also send EM noise waves back into the antenna array. These waves are coupled into other receiver channels, giving rise to a correlated noise contribution. This effect is known as noise coupling, which contributes as $\mathbf{R}_{\text {rec }}$ in a general and non-diagonal noise correlation matrix represented as:

$\boldsymbol{\Sigma}_{n}^{\prime}=\mathbf{R}_{\mathrm{sp}}+\mathbf{R}_{\mathrm{sky}}+\mathbf{R}_{\mathrm{rec}}$

where $\mathbf{R}_{\mathrm{sp}}$ is the spillover noise correlation matrix that is usually negligible as compared with $\mathbf{R}_{\text {sky }}$ which is the sky noise contribution. Crosstalk in the back-end adds another baseline-specific correlated noise term, which we disregard here. The general data model for the visibility matrix becomes:

$$
\mathbf{R}=\mathbf{G M A} \boldsymbol{\Sigma}_{\mathrm{s}} \mathbf{A}^{\mathrm{H}} \mathbf{M}^{\mathrm{H}} \mathbf{G}^{\mathrm{H}}+\boldsymbol{\Sigma}_{n}^{\prime} .
$$

We note that $\boldsymbol{\Sigma}_{n}^{\prime}$ is not a diagonal matrix, unlike $\boldsymbol{\Sigma}_{n}$. It can be shown that noise from each station element still has a normal distribution in view of the large number of samples and according to the central limit theorem. Therefore, the off-diagonal elements of $\boldsymbol{\Sigma}_{n}^{\prime}$ that appear in Eq. (10), have a Wishart distribution.

Although the noise model in Eq. (7) has only been presented only in the literature for phased array feeds (PAFs), for example by Jeffs et al. (2008) and Ivashina et al. (2011), it can be used as a generic noise model for any antenna system (Maaskant 2010). It is clear, however, that depending on the antenna and LNA designs, the electrical characteristics of the array, and both the sparsity and density of the station element layout, the strength of mutual coupling varies. Thus, the term $\mathbf{R}_{\text {rec }}$ may be replaced by uncorrelated receiver noise in this definition.

\subsection{Model-based calibration method}

The model-based calibration problem was formulated as a least squares minimization problem by Wijnholds \& van der Veen (2009a). Here, we rewrite it using the refined data model given in Eq. (8)

$$
\left\{\hat{\boldsymbol{g}}, \hat{\boldsymbol{\sigma}}_{n}\right\}=\operatorname{argmin}_{\boldsymbol{g}, \boldsymbol{\sigma}_{n}}\left\|\mathbf{G M A} \boldsymbol{\Sigma}_{\mathbf{s}} \mathbf{A}^{\mathrm{H}} \mathbf{M}^{\mathrm{H}} \mathbf{G}^{\mathrm{H}}+\boldsymbol{\Sigma}_{n}^{\prime}-\hat{\mathbf{R}}\right\|_{F}^{2} .
$$

This estimates the noise and complex gain of each receiver element using the measured visibility, $\hat{\mathbf{R}}$ and the modeled visibility $\mathbf{G M A} \boldsymbol{\Sigma}_{\mathrm{s}} \mathbf{A}^{\mathrm{H}} \mathbf{M}^{\mathrm{H}} \mathbf{G}^{\mathrm{H}}+\boldsymbol{\Sigma}_{n}^{\prime}$, where $\boldsymbol{\Sigma}_{\mathrm{s}}$ and $\mathbf{A}$ are assumed to be known. We can calculate them, if we specify the time of observation, the telescope geometry and the known source parameters. In the presence of the coupling effects ( $\mathbf{M}$ and $\boldsymbol{\Sigma}_{n}^{\prime}$ ), estimation results are biased, unless either an accurate beam model of each individual element is provided or the matrix $\mathbf{M}$ is known. In this case, one can handle the effect of the correlated noise using a WALS approach (Wijnholds \& van der Veen 2009b).

Figure 2 shows a sky map scanned by HBA tiles. One can see the Galactic plane owing to the many short baselines and the Sun as the dominant radio source. Given the beam models of the station elements, the WALS method treats the extended structure as correlated noise and estimates the parameters of interest. However, the model-based methods are in general iterative methods that usually converge after several iterations.

\subsection{Redundancy calibration method}

The basic assumption of redundancy calibration is that the redundant baselines sample the same Fourier component of the sky $\left(\mathbf{A} \boldsymbol{\Sigma}_{\mathrm{s}} \mathbf{A}^{\mathrm{H}}\right)$. This assumption holds if the array response vectors of the redundant baselines are the same i.e. the element beams are identical. We therefore begin with the most general data model given in Eq. (8) to understand the limitations of this method for a phased-array station.

To build up the system of equations for the redundancy calibration algorithm, we represent an off-diagonal element of $\mathbf{R}$ in Eq. (8) as

$R_{i j}=g_{i} g_{j}^{*}\left[\mathbf{M} \mathbf{A} \Sigma_{\mathbf{s}} \mathbf{A}^{\mathrm{H}} \mathbf{M}^{\mathrm{H}}\right]_{i j}+\Sigma_{n_{i j}}^{\prime}$.

We note that correlator offsets could contribute as additive corrupting factors in $\Sigma_{n_{i j}}^{\prime}$. Since $\Sigma_{\mathrm{s}}$ in Sect. 2.1 is a diagonal matrix, one can expand Eq. (10) to

$$
\begin{aligned}
R_{i j}=g_{i} g_{j}^{*} M_{i i} M_{j j}^{*} \overbrace{\sum_{q=1}^{q} A_{i q} \sigma_{q} A_{j q}^{*}}^{\text {true visibility }} \\
+\underbrace{g_{i} g_{j}^{*} \sum_{q=1}^{q}\left(\sum_{\left.\substack{p_{1}=1 \\
p_{1} \neq i} \sum_{p_{2} \neq j}^{p} \sum_{i p_{1}}^{p} M_{j p_{2}}^{*} A_{p_{1} q} A_{p_{2} q}^{*} \sigma_{q}\right)}^{p}\right)}_{\text {additive term due to mutual coupling }}+\Sigma_{n_{i j}}^{\prime} .
\end{aligned}
$$


This shows that the mutual coupling produces baselinedependent multiplicative and additive terms by influencing signals directly and noise indirectly ( $\mathbf{R}_{\mathrm{rec}}$ in Eq. (7)). This violates the fundamental assumption of redundancy i.e. we do not observe redundant visibilities on physically redundant baselines (see Fig. 4, top).

Without loss of generality, $M_{i i} M_{j j}^{*}=1$ (or they can be absorbed in the gains). To establish an analogy between Eq. (11) and the redundancy method formalism in Wieringa (1991), we define the term

$e_{i j}=g_{i} g_{j}^{*} \sum_{q=1}^{q}\left(\sum_{\substack{p_{1}=1 \\ p_{1} \neq i}}^{p} \sum_{\substack{p_{2}=1 \\ p_{2} \neq j}}^{p} M_{i p_{1}} M_{j p_{2}}^{*} A_{p_{1} q} A_{p_{2} q}^{*} \sigma_{q}\right)+\Sigma_{n, i j}^{\prime}$

and rewrite Eq. (11) as:

$R_{i j}^{\mathrm{obs}}=R_{i j}^{\mathrm{true}} g_{i} g_{j}^{*}+e_{i j}$

or

$R_{i j}^{\mathrm{obs}}=R_{i j}^{\mathrm{true}} g_{i} g_{j}^{*} \underbrace{\left(1+\frac{e_{i j}}{R_{i j}^{\text {true }} g_{i} g_{j}^{*}}\right)}_{w_{i j}}$

where $R_{i j}^{\text {obs }}$ and $R_{i j}^{\text {true }}$ are the observed and the theoretical redundant visibilities, respectively, $g_{i}$ and $g_{j}$ are the element complex gains and $w_{i j}$ can be defined as a baseline-dependent error that affects the accuracy of the calibration results.

We take the natural logarithm of both sides of Eq. (14) to obtain

$\ln R_{i j}^{\mathrm{obs}}=\ln \left|\gamma_{i}\right|+\ln \left|\gamma_{j}\right|+i\left(\phi_{j}-\phi_{i}\right)+\ln R_{i j}^{\mathrm{true}}+\ln \left(w_{i j}\right)$.

In the absence of mutual coupling, the first term of $e_{i j}$ drops and the second term reduces the contribution of $R_{\text {rec }}$. The only correlated contributing terms come from $R_{\text {sky }}$ and $R_{\mathrm{sp}}$, which are negligible depending on the signal-to-noise ratio $(\mathrm{S} / \mathrm{N})$ of the observation. We assume that we have such a case where we can ignore $e_{i j}$. We equate the amplitude and the phase values to decouple the system of equations for the phases and the amplitudes

$\ln \left|R_{i j}^{\mathrm{obs}}\right|=\gamma_{i}^{\prime}+\gamma_{j}^{\prime}+\ln \left|R_{i j}^{\mathrm{true}}\right|$

$\psi_{i j}^{\mathrm{obs}}=\phi_{j}-\phi_{i}+\psi_{i j}^{\mathrm{true}}$,

where $\left|R_{i j}\right|$ and $\psi_{i j}$ are the amplitude and the phase of a complex visibility. Since we have to specify the absolute flux level, we set $\Sigma \gamma_{i}^{\prime}=0$.

We also have to constrain the element phase. We can enforce this constraint by specifying that the average phase for all elements is zero

$\Sigma \phi_{i}=0$.

Furthermore, there may also be an arbitrary linear phase slope over the array. This phase slope corresponds to a position shift of the field. This arises because a redundancy solution does not provide an absolute position. This can be absorbed into either the true visibilities or the element phases. For a two-dimensional array, we constrain $x$ and $y$ in the same manner as

$\sum_{i=1}^{p} \phi_{i} x_{i}=0$

$\sum_{i=1}^{p} \phi_{i} y_{i}=0$ where $x_{i}$ and $y_{i}$ are the $(x, y)$ coordinates of the array elements. Equations (16)-(21) formulate the redundancy calibration method as two overdetermined systems of linear equations for phases and amplitudes that can be solved in a single-step least squares solution. For instance, the phase estimator can be symbolized as $\mathbf{E}_{\mathrm{ph}} \boldsymbol{\theta}=\boldsymbol{\Psi}^{\mathrm{obs}}$ and solved using the pseudo-inverse $\hat{\boldsymbol{\theta}}=\left[\mathbf{E}_{\mathrm{ph}}^{\mathrm{T}} \mathbf{E}_{\mathrm{ph}}\right]^{-1} \mathbf{E}_{\mathrm{ph}}^{\mathrm{T}} \boldsymbol{\Psi}^{\mathrm{obs}}$,

where $\boldsymbol{\theta}=\left[\phi_{1}, \phi_{2}, \ldots, \phi_{p}, \psi_{1}^{\text {true }}, \psi_{2}^{\text {true }}, \ldots, \psi_{m}^{\text {true }}\right]$ is the vector of parameters to be estimated, $m$ is the number of distinct redundant baselines, $\mathbf{E}_{\mathrm{ph}}$ is the coefficient matrix, and $\boldsymbol{\Psi}^{\mathrm{obs}}$ is the vector of the observed redundant phases and right sides of Eqs. (19)-(21). Setting a phase reference for element phases, is done after the phase estimation.

We note that we came to the solution in Eq. (22), because we ignored the baseline-dependent noise. In its presence, our problem takes the form of $\mathbf{E}_{\mathrm{ph}} \boldsymbol{\theta}+\boldsymbol{\beta}=\boldsymbol{\Psi}^{\mathrm{obs}}$ (expanded in Eq. (24)) The estimated parameters will deviate as

$\boldsymbol{\varepsilon} \equiv \boldsymbol{\theta}-\hat{\boldsymbol{\theta}}=\left[\mathbf{E}_{\mathrm{ph}}^{\mathrm{T}} \mathbf{E}_{\mathrm{ph}}\right]^{-1} \mathbf{E}_{\mathrm{ph}}^{\mathrm{T}} \boldsymbol{\beta}$,

where $\boldsymbol{\beta}=\operatorname{vec}\left(\angle\left(\ln \left(w_{i j}\right)\right)\right)$. If the array mutual coupling is significant, the redundancy method is not be a reliable estimator. In the case of a weakly coupled array, the vector $\beta$ still carries the correlated noise produced by $R_{\mathrm{sp}}$ and $R_{\text {sky }}$. Assuming that each has a Wishart distribution in Eq. (12), their statistical distribution changes in vector $\beta$. This was taken into account in deriving the results in Sect. 3.2.

Since the created systems of equations are highly sparse, they are computationally fast. Most importantly, they are independent of a sky model but their accuracy is affected by the $\mathrm{S} / \mathrm{N}$ of the observed sky as discussed by Liu et al. (2010). They require identical beams of station elements and a regular arrangement of antennas to provide a sufficient number of redundant baselines. One can then, estimate the element complex gains in a single step.

\section{Implementation of the redundancy calibration using real and simulated HBA data}

\subsection{Verification of the fundamental assumption of redundancy}

As mentioned earlier, redundancy translates into verifying the similarities among the element beams in a station. We checked this for a 24-tile HBA station similar to the one shown in Fig. 1 using Computationally Advanced and Efficient Simulator for ARrays (CAESAR; Maaskant et al. 2008; Maaskant \& Yang 2006). The numerical computation of the EM simulation was done using the Characteristic Basis Function Method (CBFM), which is the most numerically efficient and accurate method available for large scattering problems (Prakash \& Mittra 2003; Yeo et al. 2003). Figure 3 presents the simulation results when the beams are formed toward the local zenith. Each subplot presents the beam patterns of 24 tiles for a particular frequency in the $\phi=0$ plane. One can see that owing to the different mutual coupling environment, each tile has a slightly different beam pattern. The mutual coupling effect is sufficiently small not to disturb the main beam but only the far sidelobes which are at least $12 \mathrm{~dB}$ weaker than the main lobe. However, it is not a favorable condition in general for redundancy calibration. On the basis of this simulation, we expect to observe non-redundant visibilities on redundant baselines when a strong source falls in the sidelobes. This was confirmed by the real observation presented 

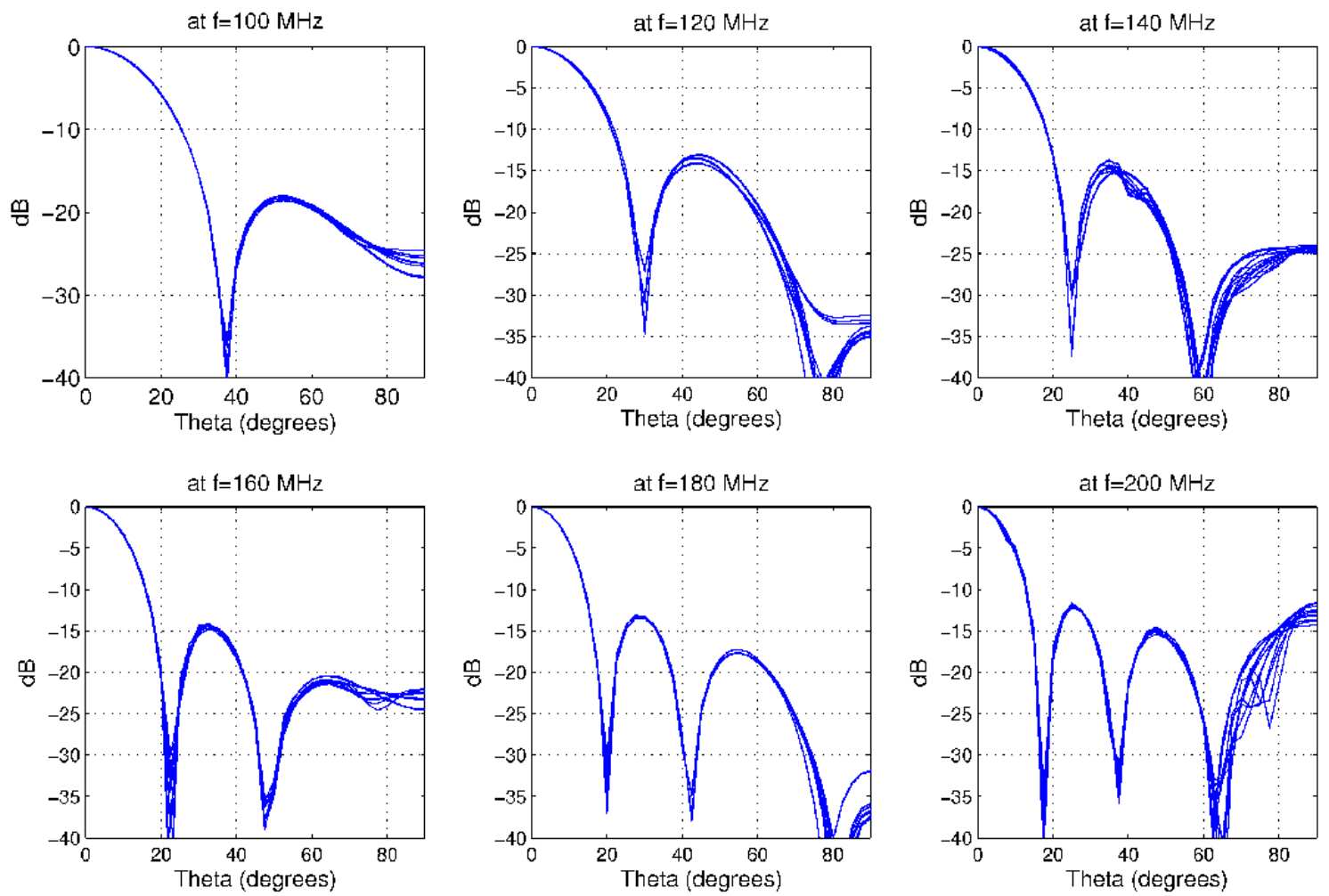

Fig. 3. Simulated beam pattern of the tiles in a 24-tile HBA station using CAESAR. Each subplot presents the beam patterns of 24 tiles in a particular frequency in $\phi=0$ plane. The beams are formed toward the local zenith. The dissimilarity between the sidelobes is caused by the different mutual coupling environments of each tile.

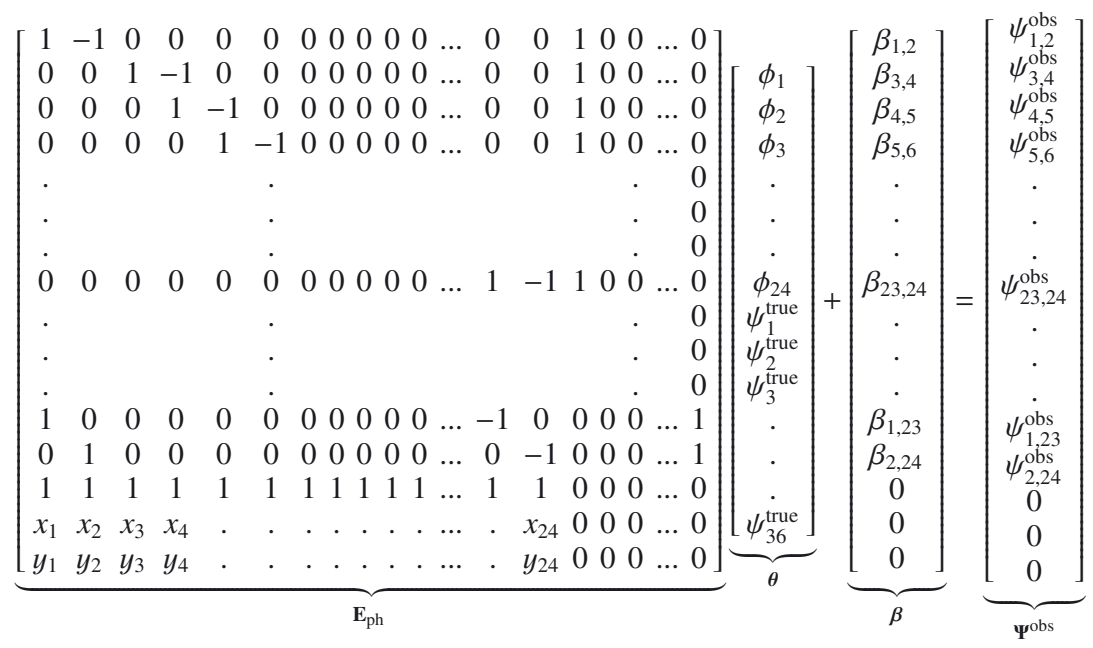

in Fig. 4, top panel. In this observation, owing to the absence of a strong source in the main beam, the non-identical sidelobes have the chance to play a significant role in disturbing the redundancy while in the observation shown in Fig. 4, bottom panel, one strong source in the main beam seems to be sufficient to dominate the influence of other possible strong sources observed in the direction of the non-identical sidelobes. There is of course a different contribution from the correlated background noise or sky noise $\left(\mathbf{R}_{\text {sky }}\right)$ in Fig. 4, top and bottom panels.

Figure 4 presents two data sets obtained to verify the use of redundancy in a HBA station. The observations were made on 5 September 2009. The tile beams in a HBA station (in the LOFAR core, known as CS302) were formed toward the local zenith. We let the sky drift over the field of view (FoV) of the station. We captured the station visibilities approximately every
10 min (integration time of one second per frequency channel for 512 frequency channels). The bandwidth of the frequency channels is $195 \mathrm{kHz}$ and the frequency range is $100-200 \mathrm{MHz}$. On the right panel, the local sky viewed from CS302 is presented to show the sources that have contributed to the visibilities shown in the left panel.

Since the mutual coupling environment changes at lower elevations, dissimilarities between the main beams are consequently found up to 1-2 dB. Real observations have shown less disturbance in the main beam at lower elevations (see Sect. 3.2 and Appendix A).

On the basis of the EM simulation by CAESAR (Fig. 3) and real observations (Fig. 4), we conclude that the best case scenario for redundancy calibration of an HBA station is to have a strong source in the main lobe when the beams are 

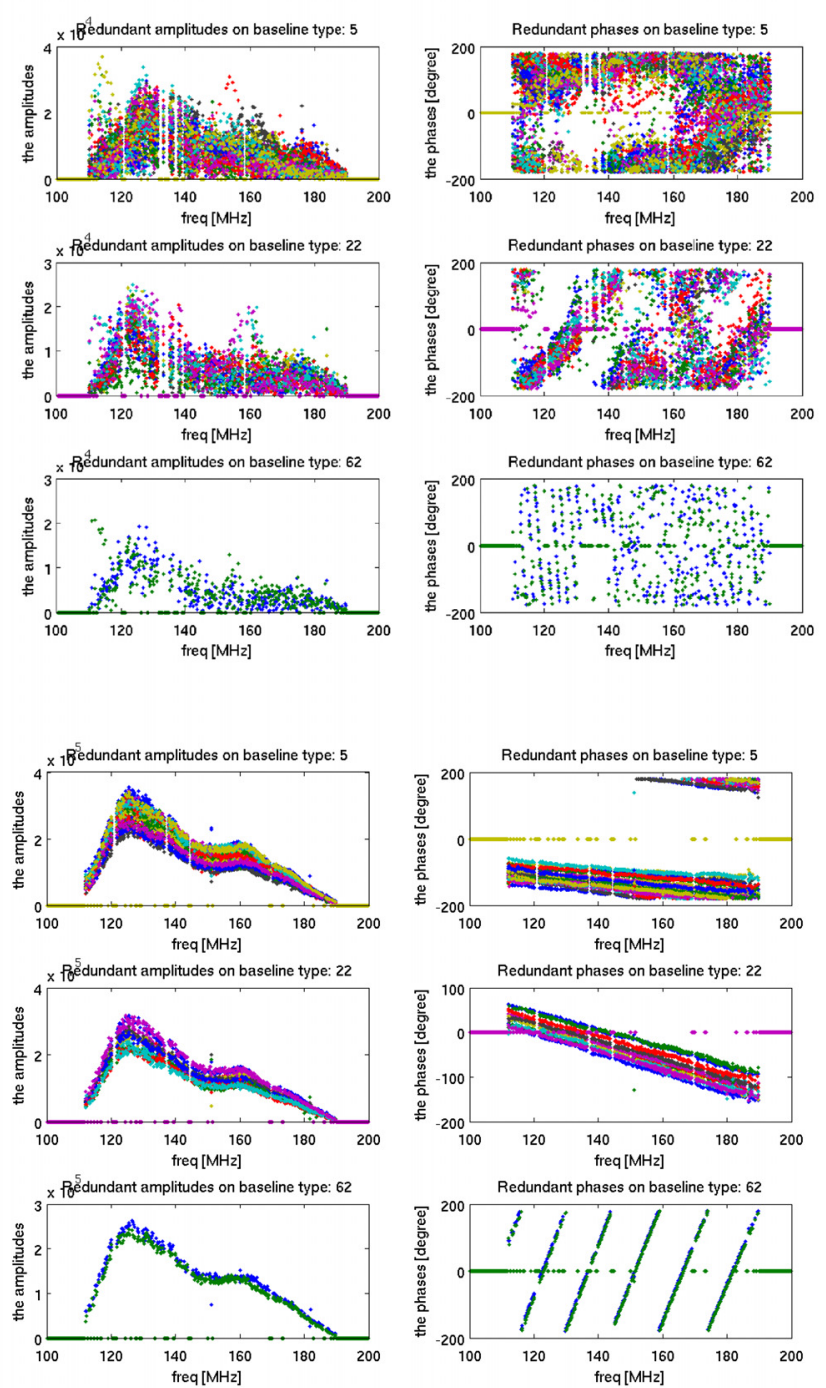

the local sky in view of CS302, at: 05-Sep-2009 13:13:48

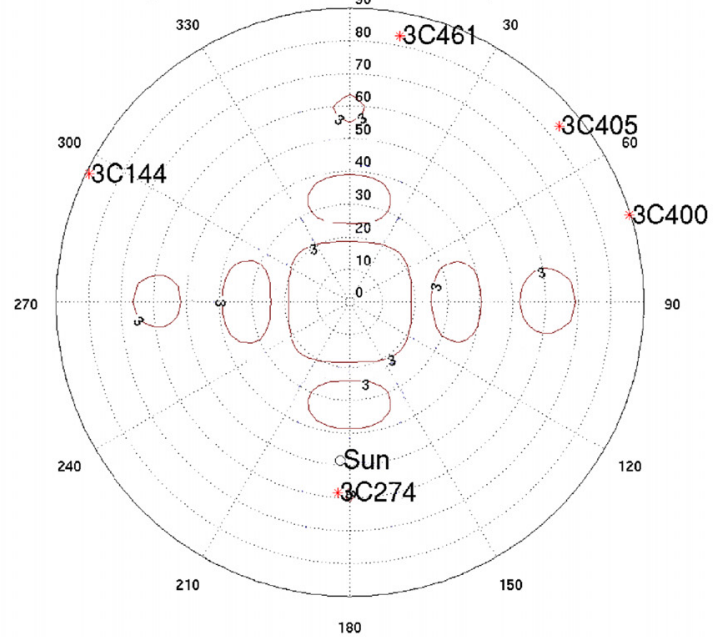

the local sky in view of $\mathrm{CS}_{0.20}$ at: 05-Sep-2009 23:02:01

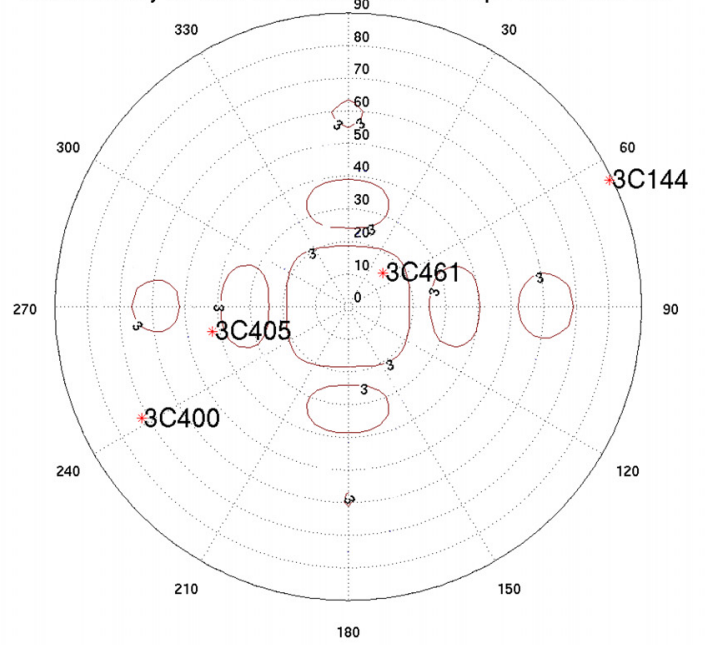

Fig. 4. An observation performed with a HBA station on September 5, 2009 at 13:13:48 UTC in which redundancy calibration fails (top), as well as an observation with the same station at 23:02:01 UTC in which the redundancy calibration is successful (bottom). The left panels show the measured visibilities for three distinct types of redundant baselines. The right panels show the local sky model at the time of observation, where the strongest sources on the sky are superimposed on a contour plot of the element beam pattern at $170 \mathrm{MHz}$.

identical. However, the dissimilarities of the sidelobes introduce non-redundancy or systematic errors that cannot be eliminated by any statistical method or a longer integration time. Therefore, we must investigate whether the contribution of other strong sources, which may fall in the non-identical sidelobes, is significant. To quantify this systematic error in the visibility measured on a given baseline, we present the following example. At 21:29:04 UTC on 24 November 2009, at an HBA station called RS208 located at $\left(\right.$ lon $=6.9196^{\circ}$, lat $\left.=52.6699^{\circ}\right)$, four strong sources were in the FoV, as shown in Fig. 5. In Fig. 5, the tile beams were formed toward 3C 461 (CasA), which was thus at the phase center. The sources 3C 405 (CygA), 3C 400, and 3C 144 (Tau A) have fallen in the sidelobes. The tile beams and their standard deviation at $120 \mathrm{MHz}$, at these sources are also depicted in Fig. 5. The total complex visibility observed on a certain baseline, $\mathbf{D}_{\lambda}$ is computed as

$\mathbf{R}^{\mathrm{obs}}=\left|R^{\mathrm{obs}}\right| \mathrm{e}^{j \psi^{\mathrm{obs}}}=\int_{\mathrm{s}} \Lambda(\sigma) I(\sigma) \mathrm{e}^{-j 2 \pi \mathbf{D}_{\lambda} \cdot \sigma} \mathrm{d} \Omega$ where $\Lambda(\sigma) \equiv A(\sigma) / A_{0}$ is the normalized tile reception pattern at $\sigma, A_{0}$ is the response at the beam center and $I(\sigma)$ is the source flux. Since there are four dominant strong sources in the FoV, we assume that the integral can be replaced by a summation. The complex plane in Fig. 6 right panel, shows the summations of the visibility vectors as they were observed through hypothetically identical sidelobes, $\mathbf{R}_{\text {total }}$ (solid line), as well as their summation when they were attenuated differently by the actual non-identical sidelobes, $\mathbf{R}_{\text {total }}^{\prime}$ (dotted line). We computed the systematic errors in the phase and the amplitude of the visibility by assuming the complex vector to be $\mathbf{R}_{\text {total }}=\mathbf{R}_{3 \mathrm{C} 461}+$ $\mathbf{R}_{3 \mathrm{C} 144}+\mathbf{R}_{3 \mathrm{C} 405}+\mathbf{R}_{3 \mathrm{C} 400}$ using Eq. (25). For this, we choose the baseline type given in Fig. 8, rightmost panel (D) and frequency $f=120 \mathrm{MHz}$ for $\mathbf{D}_{\lambda}=\mathbf{D} / \lambda$, where $\lambda$ is the wavelength. We set the average beam pattern of all 48 tiles as the identical beam for the term $A(\sigma)$ whose values are shown in Fig. 5 at different source locations, as the quantity beam level. We use $\Lambda(\sigma)=$ $A(\sigma) / A\left(\sigma_{3 \mathrm{C} 461}\right)$. The source fluxes, $I(\sigma)$ are given in Table 1. We also compute $\mathbf{R}_{\text {total }}^{\prime}=\mathbf{R}_{3 \mathrm{C} 461}^{\prime}+\mathbf{R}_{3 \mathrm{C} 144}^{\prime}+\mathbf{R}_{3 \mathrm{C} 405}^{\prime}+\mathbf{R}_{3 \mathrm{C} 400}^{\prime}$. 


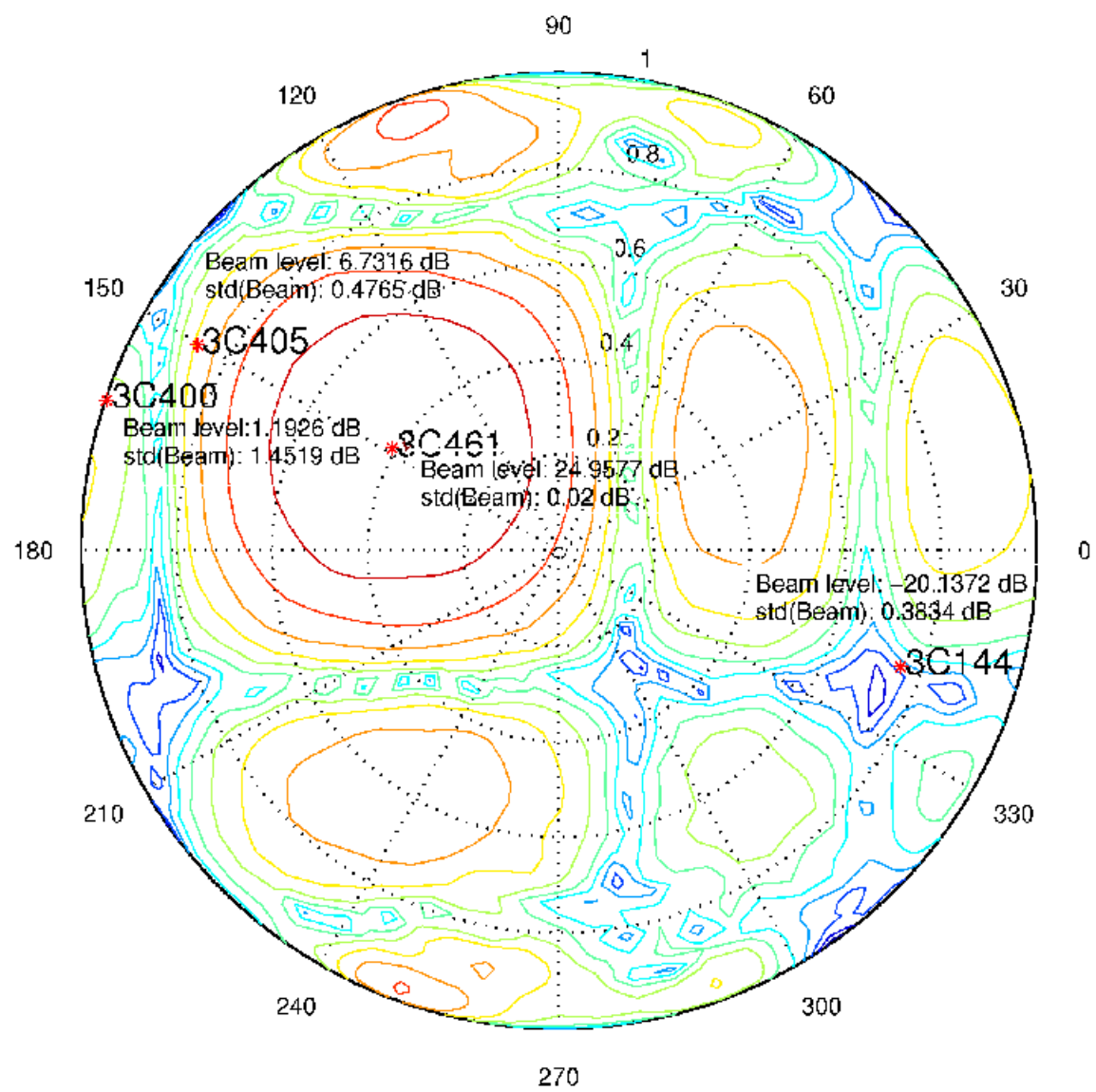

Fig. 5. A-team radio sources in the FoV of an HBA station called RS208 on 24 November 2009 at 21:29:04 UTC. Tile beams are streered toward 3C 461 to provide the most redundant visibilities. The values of the average reception pattern of 48 tiles, $A(\sigma)$, and their standard deviation, $\operatorname{std}(A(\sigma))$ at $120 \mathrm{MHz}$ have also been indicated at other source locations, $\sigma$.

The computation settings are as before except that $A(\sigma)$ is not identical this time, but deviated from the average beam pattern by the standard deviation values shown in Fig. 5 at different source locations, as the quantity $\operatorname{std}($ Beam). The bias introduced to the amplitude and the phase of $\mathbf{R}_{\text {total }}$ as compared with $\mathbf{R}_{\text {total }}$ are presented in the first row of Table 1 .

We steer the beam toward each available strong source and repeat the calculation to predict the systematic error due to the other sources in the sidelobes with different standard deviations. The results are presented in Table 1 . We can conclude that tracking CasA provides the best redundancy in the observed visibilities.

The plot of the residuals for the corrected redundant visibilities shown in Fig. 8 confirms the results in Table 1. Figure 8 reveals the non-redundancy in the measured visibilities (in terms of amplitudes and phases). In Table 1, we predict the same quantities, which are caused by non-identical sidelobes. There is a few percent of discrepancy between the predicted residuals and their actual values in our observation. This can be explained by our simplifying assumption about the number of strong sources in the FoV. However, Table 1 can play an instrumental role for station calibration.

\subsection{Redundancy calibration performance}

To study the redundancy calibration performance, we tracked CasA on 24 November 2009 from 15:25:43 UTC until
22:12:19 UTC. Running a redundancy calibration on 48 captured data sets during this observation, provided very stable results for the receiver complex gains over time. This is an indication of the system stability and a working calibration routine, which operated approximately every $10 \mathrm{~min}$. We compare the variance in the estimated complex gains over 48 runs of redundancy calibration with the CRB in Fig. 7. These quantities for the amplitudes and the phases are presented in two separate plots, as their estimators were decoupled in Sect. 2.3. The CRB (or the theoretical minimum variance) and the actual variance in the estimated parameters over time are in good agreement. The small difference between them can be explained by their not having exactly the same $\mathrm{S} / \mathrm{N}$ from one observation to another during our survey from 15:25:43 UTC until 22:12:19 UTC, while the predicted $\mathrm{S} / \mathrm{N}$ for this observation is $S / N \simeq 0.75$, following the analysis of Wijnholds \& van Cappellen (2011). This predicted value was used to compute the presented CRB in Fig. 7.

We also studied the residuals for the corrected redundant visibilities. Figure 8 shows an example of the residuals in both the amplitudes and phases of the corrected visibilities on a distinct type of redundant baseline. The snapshot is captured at 21:29:04 UTC, when CasA is at high elevation. The first row compares the results after redundancy calibration to those after the model-based calibration in the second row. The type of redundant baseline is depicted on the station configuration in the rightmost panel. The integration time is one second per frequency channel. More plots of the residuals from the same observation are presented in Fig. A.1. By comparing them, one 

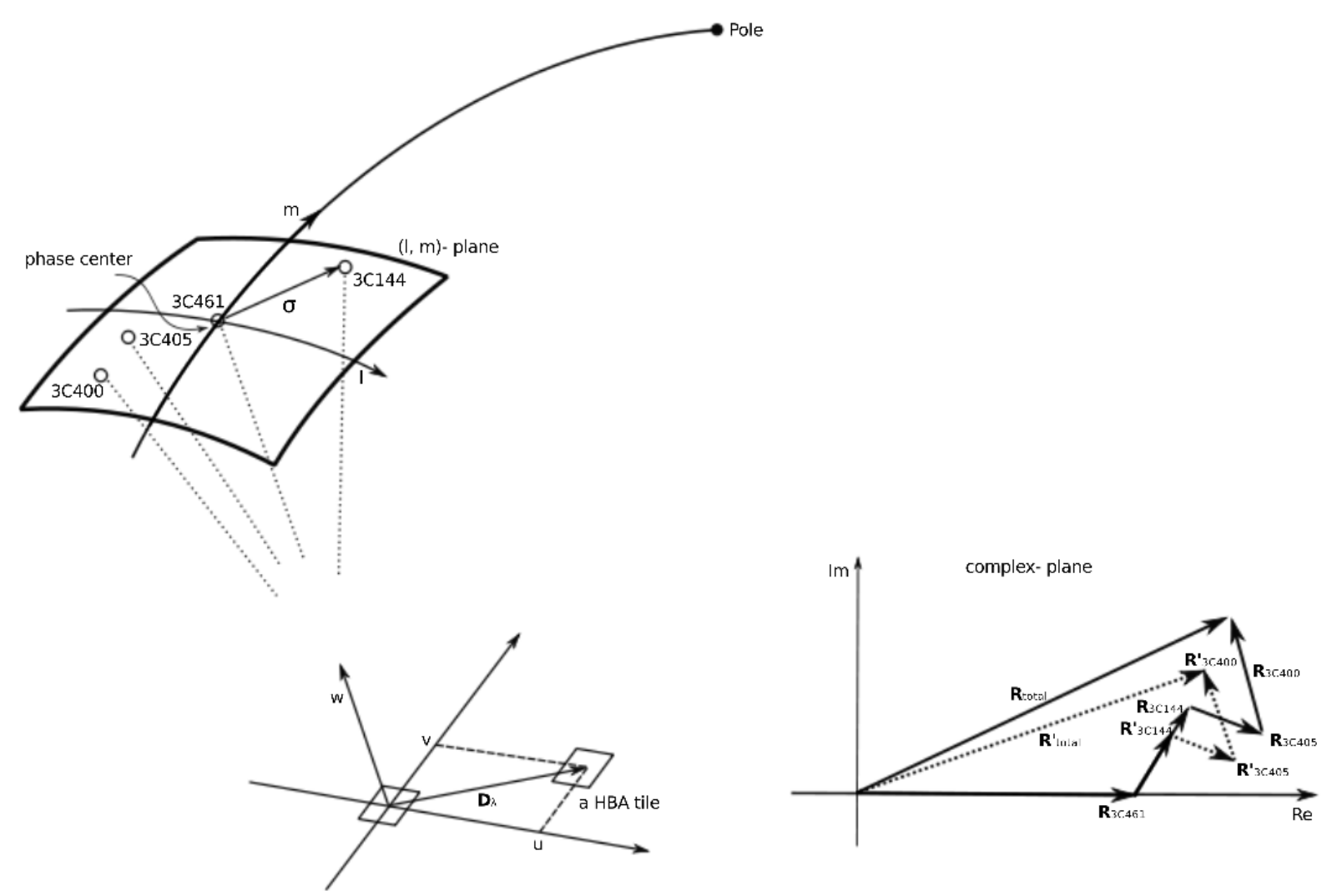

Fig. 6. Total complex visibility on a certain baseline due to the contribution of all strong sources shown in Fig. 5. The ones that contribute through non-identical sidelobes introduce non-redundancy or systematic errors into the total redundant visibility. This has been demonstrated in a complex plane on the right. The solid line shows the visibility vectors as they were observed through hypothetically identical sidelobes. The dotted line shows the visibility vectors when they are attenuated differently by the actual non-identical sidelobes.

Table 1. A-team radio sources in the FoV of RS208, on 24 of November 2009 at 21:29:04 UTC and the predicted levels of systematic errors in the amplitudes and the phases of the redundant visibility due to the contribution of other strong sources through non-identical sidelobes.

\begin{tabular}{lccc}
\hline \hline Calibrator source & Source flux [Jy] & Normalized error in amplitudes & Error in phases [rad] \\
\hline 3C 461 (CasA) & 8609 & 0.07 & 0.06 \\
3C 405 (CygA) & 8100 & 0.20 & 0.09 \\
3C 400 & 540 & 0.93 & 0.01 \\
3C 144 (TauA) & 1420 & 0.84 & 0.28 \\
\hline
\end{tabular}

Notes. The baselines given in Fig. 8, rightmost panel and frequency, $f=120 \mathrm{MHz}$ were chosen for the computations.

may note that the baseline length does not make a significant difference in the residuals. The residuals are on the order of $2-5 \%$ in both phases and amplitudes. Because a strong source such as CasA in the field of view dominates the effect of any possible correlated noise, this can be explained by not having $100 \%$ identical main beams or the corruption due to the nonidentical sidelobes (see Table 1). However, these results are satisfactory. Moreover, Fig. 7 showed that the estimated complex gains, which are our parameters of interest in a station calibration, are very stable. This is due to the constraints on them in the formalism of the redundancy calibration.

Using the data captured at 15:25:43 UTC, when CasA is at low elevation, reveals slightly larger residuals in the order of $5-10 \%$ (see Fig. A.2). This is due to a different mutual coupling environment which leads to less identical beams in either the main lobe or the sidelobes.

The residuals also show that the two calibration methods perform almost equally well, although the redundancy method behaves more consistently. The model-based calibration has slightly larger residuals on short baselines, e.g. up to $\sim 0-1 \%$ in phases and $\sim 0-2 \%$ in amplitudes, whereas it shows similar residuals as the redundancy calibration on long baselines. Extended structures such as the Galactic plane or north polar spur, are captured on short baselines. Modeling them is computationally expensive and somehow impractical. Therefore, in the model-based method, one has to discard the visibilities measured on short baselines to simplify the measured sky for a corresponding simple sky model, while the redundancy method is sky-model-independent. Experiment has shown that discarding the equations of short baselines in the redundancy calibration routine, will not improve its results significantly.

Moreover, the model-based methods are sensitive to RFI sources, as their presence confuses the sky model. Since the redundancy calibration is independent of the sky model, it is less sensitive to RFI. One may note that more frequency channels had to be flagged in the model-based calibration. 
P. Noorishad et al.: Redundancy calibration of phased-array stations
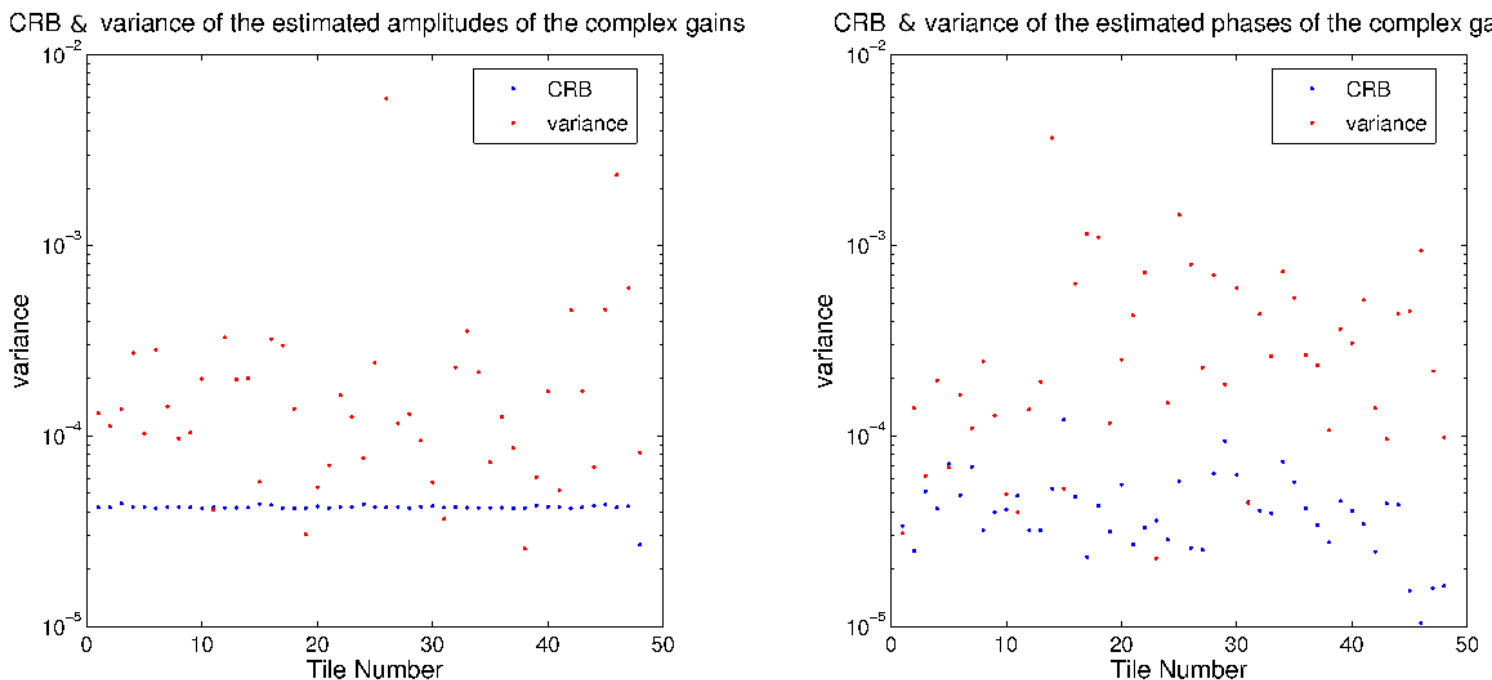

Fig. 7. Theoretical minimum variances in the estimated amplitudes and phases of the complex gains or CRB compared with their actual variance. These quantities for the amplitudes and the phases are presented in two separate plots as their estimators were decoupled in Sect. 2.3 .
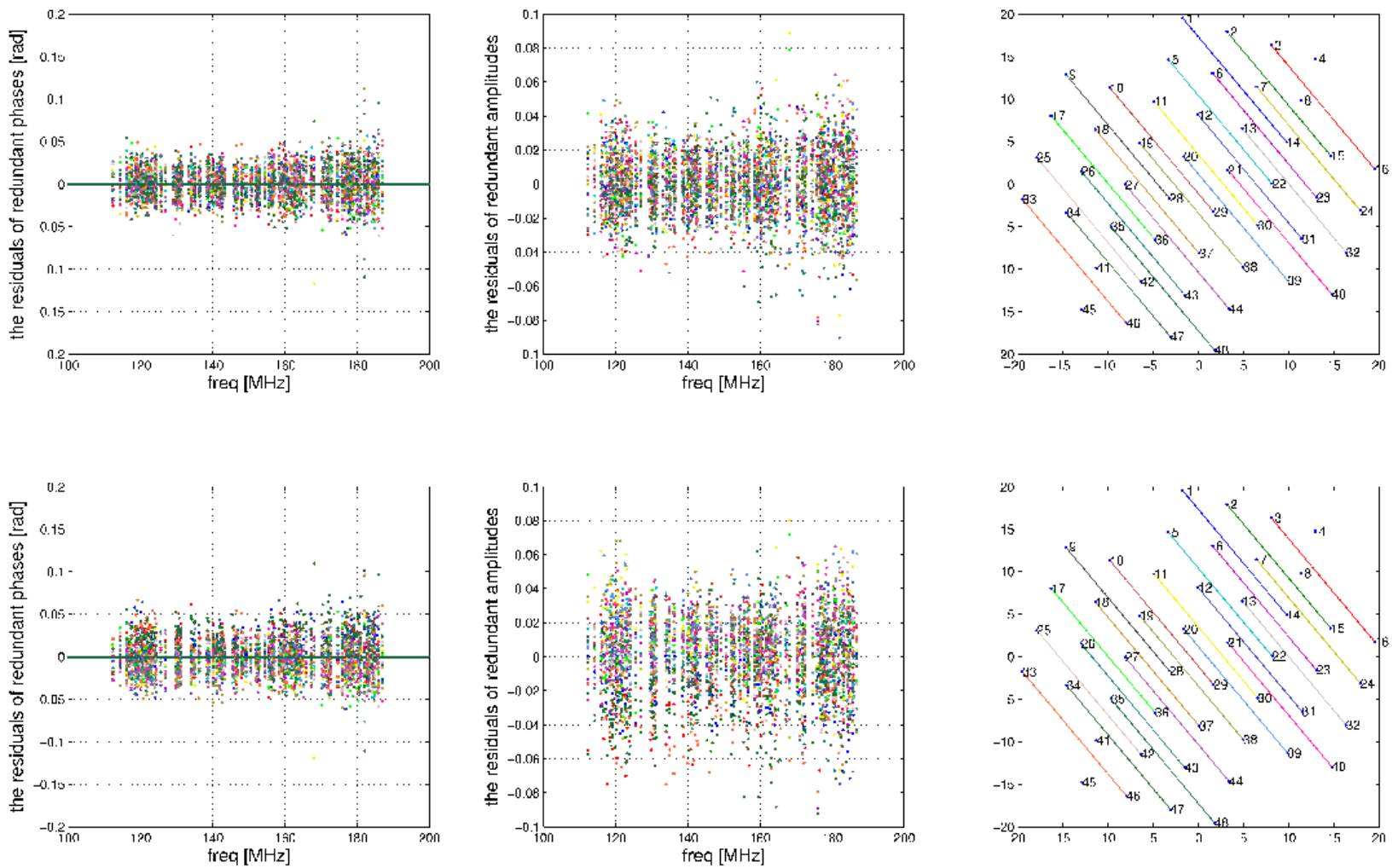

Fig. 8. Plots of the residuals for corrected redundant visibilities in terms of phases and amplitudes on a given set of redundant baselines. The first row shows the results after redundancy calibration. The second row shows the result after model-based calibration. The data is taken from the observation done on 24 November 2009 at 21:29:04 UTC, when CasA is at high elevation. The station configuration of RS208 is shown in the rightmost panel. The corresponding redundant baselines to each redundant visibility are also depicted on the station layout, with the same color code.

As discussed by Liu et al. (2010), redundancy calibration quality depends on the $\mathrm{S} / \mathrm{N}$ of the observed sky, although it is independent of a sky model. Liu et al. (2010) showed that the estimated parameters are affected differently in the presence of baseline-dependent noise, assuming that it is Gaussian noise. The vector $\beta$ in Eq. (23) reveals a non-Gaussian baseline dependent noise. We have taken this into consideration in the following results. After adding different levels of a non-i.i.d.
Gaussian noise to the output vector of the array (Eq. (1)), we simulated the station visibility assuming that the station elements have identical beams. We used the station configuration of RS208 (see Fig. 8, rightmost panel). The complex gains were estimated over 100 runs of Monte-Carlo simulation for different S/N's per visibility. The variance of the error in the estimated complex gains (over simulation runs with different random noises) versus different $\mathrm{S} / \mathrm{N}$ 's per visibility is shown in 

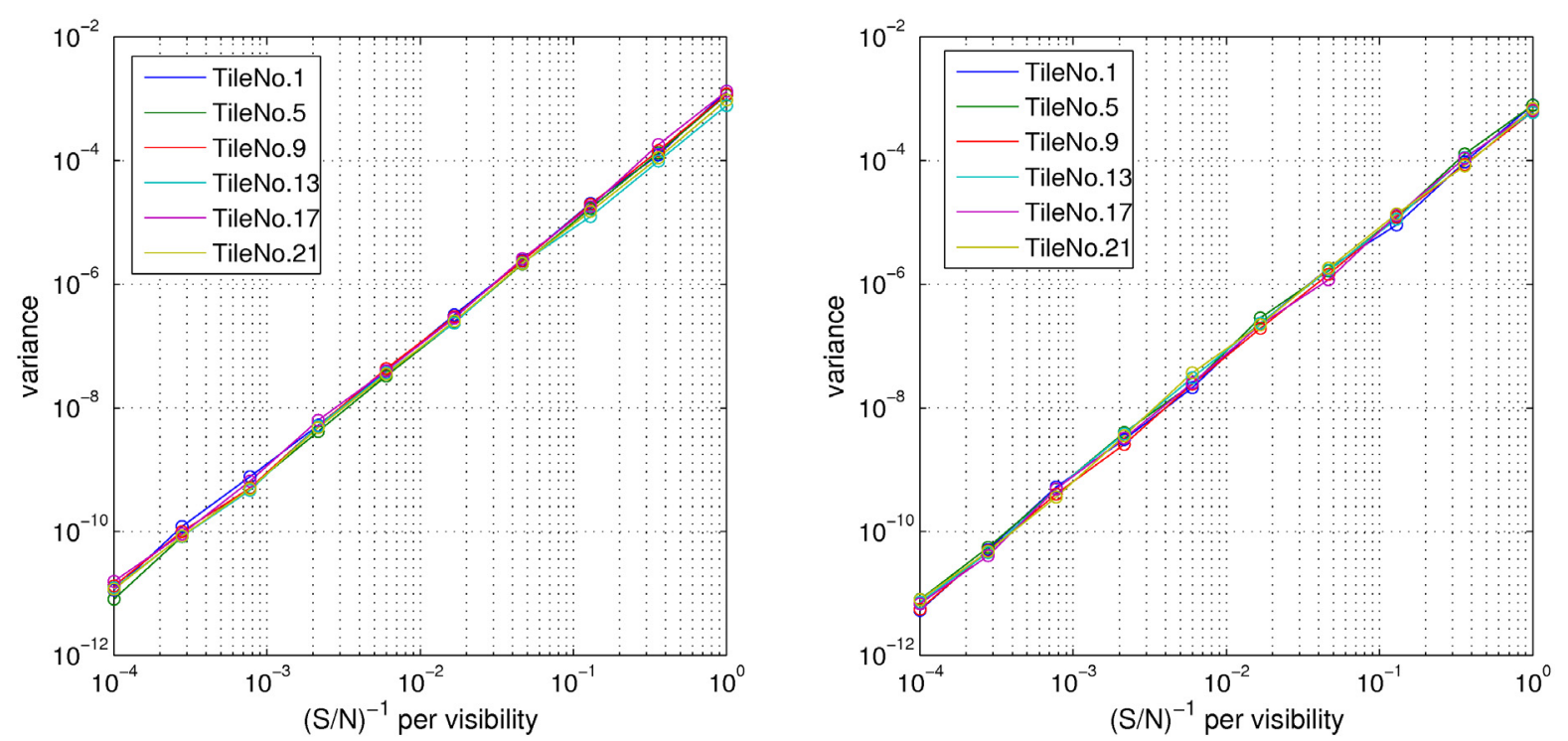

Fig. 9. The variance of the estimated complex gains in terms of amplitude $(l e f t)$ and phase (right) versus the $(S / N)^{-1}$ per visibility. Each color represents the estimated complex gain of a tile whose number is mentioned in the legend.

Fig. 9. These plots indicate that the variances in the estimated complex gains, which are our parameters of interest in a station calibration, are the same for the different tiles. This illustrates the stability of the algorithm. However, an analysis of this nature is very array-configuration-dependent, as the array configuration determines the coefficient matrices by which our estimators are defined.

\subsection{Limitations of the redundancy calibration}

The applicability of the redundancy calibration is limited by the following factors:

1. The station configuration.

2. Mutual coupling between the station elements, which leads to:

- non-identical element beams;

- the presence of baseline dependent noise.

3. The signal-to-noise ratio across the observed sky.

The station configuration can influence the suitability of redundancy in different ways. The redundancy calibration method requires a regular arrangement of station elements. In a station with $p$ elements, one needs a sufficient number of distinct types of redundant baseline to have a system of equations in which all station element gains are involved. The more redundant the station's baselines are, the less information in the measured visibilities will be missed in the computation. This is not a concern in the HBA stations, as they are highly redundant. We denote by $I$ the ratio of the number of the measured visibilities that are used in the computations, to the total number of the measured visibilities. The station configuration also determines the coefficient matrices i.e. $\mathbf{E}_{\mathrm{ph}}$ or $\mathbf{E}_{\mathrm{ampl}}$ for the phase and amplitude estimators, respectively. The condition number is a relative error magnification factor i.e. errors in the right-hand side of a linear system of equations can cause errors $\kappa\left(\mathbf{E}_{\mathrm{ph}}\right)$ times as large in the solution. Figure 10 shows three different configurations for the HBA stations in the LOFAR system. After recognizing the redundant baseline in a certain configuration, one can calculate the aforementioned quantities, which are given in Table 2. This table shows that RS208 is the most reliable configuration, from the redundancy calibration point of view. However, these quantities can be figures of merit when we design for redundancy within the stations and in terms of the arrangement of the stations within the whole array.

Configuration and element spacing in a station are usually decided based on obtaining low sidelobes of the station beam. Minimizing the mutual coupling between the station elements should be taken into consideration as other important figures of merit in a station layout for the sake of redundancy applicability. This consideration serves a twofold goal, obtaining identical element beams at least in the main beam and minimizing the receiver correlated noise $\left(\mathbf{R}_{\text {rec }}\right)$. The dissimilarities between the element beams introduces systematic errors that cannot be eliminated by either any statistical method or a longer integration time. In the presence of the baseline dependent noise, the estimated parameters will deviate from their true values as given by Eq. (23). Quantifying this deviation for a given array, requires a good understanding of the noise terms in Eq. (7), especially $\mathbf{R}_{\mathrm{rec}}$. The correlated noise terms are difficult to model analytically, Maaskant (2010). One may use a powerful and generic tool called CAESAR to compute them numerically using its electromagnetic (EM) and microwave (MW) simulators, which is indeed an ongoing project at the HBA and EMBRACE stations. Primary results from the MW-simulation for an HBA station have demonstrated a negligible contribution of $\mathbf{R}_{\mathrm{rec}}$. However, Fig. 9 indicates that whatever the effect of the calibration accuracy may be, it does not make the redundancy algorithm unstable.

\section{Discussion}

We have studied the applicability of redundancy calibration to phased-array stations for the first time. The performance of this calibration method was demonstrated using data acquired with a new telescope, LOFAR. This required new considerations to the original design of conventional arrays e.g. WSRT and VLA. We took them into account by refining the data model. Reformulating the redundancy calibration formalism using the new data model, helped us to understand its potential, limitations and the effects of non-Gaussian baseline-dependent noise on the calibration accuracy. 
P. Noorishad et al.: Redundancy calibration of phased-array stations
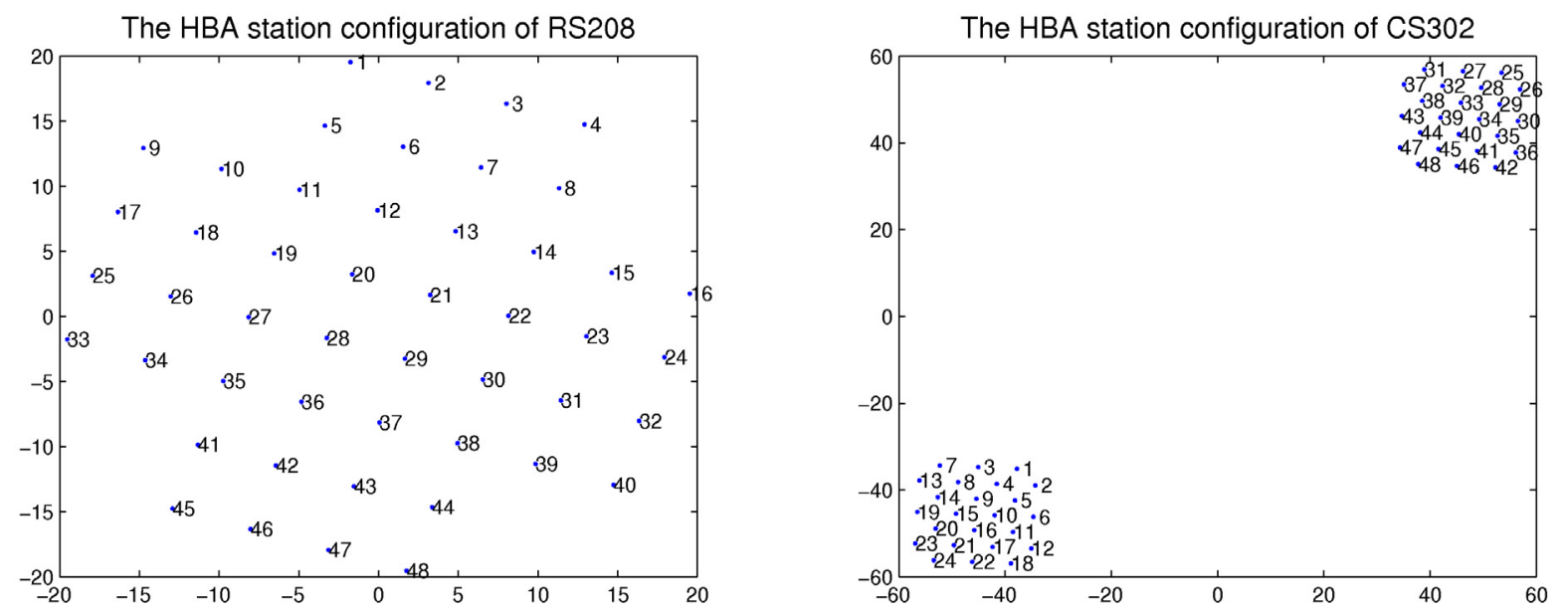

The HBA station configuration of CSO04

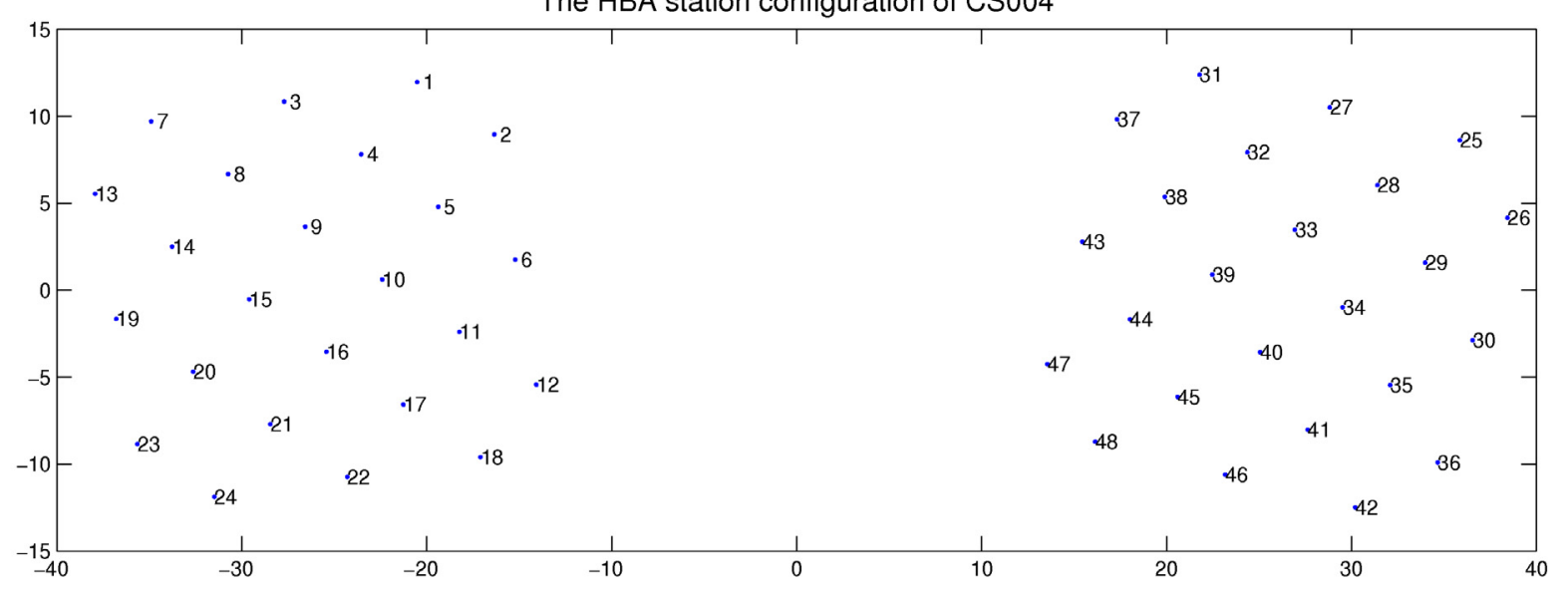

Fig. 10. Three different station configuration for HBA stations.

Table 2. Station configuration and the condition of the redundancy calibration.

\begin{tabular}{lcccccc}
\hline \hline Station Conf. & Number of distinct redundant baseline & $\operatorname{size}\left(\mathbf{E}_{\mathrm{ph}}\right)$ & $\operatorname{size}\left(\mathbf{E}_{\mathrm{ampl}}\right)$ & $\kappa\left(\mathbf{E}_{\mathrm{ph}}\right)$ & $\kappa\left(\mathbf{E}_{\mathrm{ampl}}\right)$ & $I$ \\
\hline RS208 & 84 & $1127 \times 132$ & $1125 \times 132$ & 52.72 & 9.74 & $99.65 \%$ \\
CS302 & 113 & $1123 \times 161$ & $1121 \times 161$ & $6.74 \mathrm{e} 16$ & 9.99 & $99.29 \%$ \\
CS004 & 72 & $547 \times 120$ & $545 \times 120$ & $8.42 \mathrm{e} 16$ & $1.73 \mathrm{e} 15$ & $48.22 \%$ \\
\hline
\end{tabular}

We must consider both obtaining identical beams for station elements and minimizing the correlated noise as figures of merit for the configuration and spacings between the elements within a station in Square Kilometre Array (SKA) pathfinders and the SKA itself. This is definitely necessary, regardless of the calibration method used. For the redundancy method, it is fundamental, while having identical element beams saves computational capacity and time for model-based methods.

In the plots of residuals and Table 1, we have demonstrated how non-identicalness of the beams leads to systematic errors that cannot be eliminated by any statistical methods or longer integration time. Since, identical element beams are fundamental especially for the redundancy calibration, similar EM-simulations to those presented here, are highly recommended for any array that is to be calibrated using redundancy. Moreover, redundancy turns out to be an extremely suitable diagnostic tool for recognizing and monitoring failing station elements. This is of concern in arrays with large number of elements, such as LOFAR and SKA. It is currently being used for this purpose in the LOFAR system.

Wide field science with new radio telescopes such as SKA (van Ardenne et al. 2009), demands an enormous amount of signal processing and computational capacity. This is mainly due to the large number of elements, the variable beams, and the effect of the ionosphere over a large field of view. The redundancy calibration results encourage us to seriously recommend considering redundancy in the SKA configuration, both at the station and whole array level. In large interferometers such as SKA, where the baselines are non-coplaner, one has to however, think of redundancy in UVW-space, however, not only in UV-space. Redundancy can be applied to the gain calibration of the whole array in specific regimes where the stations observe the sky, through the same ionospheric patch. It also saves computational capacity and gives a more accurate estimate of the telescope gains than the model-based gain calibration. This is of course a major step forward in achieving radio images of higher dynamic range. 


\section{Conclusion}

For the first time, we have studied the applicability and limitations of redundancy calibration in phased-array stations using both real and simulated data of a new telescope, that uses aperture array technology such as LOFAR. The results clearly show that the additional contraints provided by redundant baselines do improve the quality of the calibration and in addition provide a powerful tool for system diagnostics at different levels of the telescope phased-array hierarchy (both intra-station and interstation). The merit of redundancy in the station and full array layout and the additional advantage for diagnostics are clearly demonstrated by this study. We therefore strongly recommend to ensure redundancy in both the station layout and the array configuration of future aperture arrays, in particular the SKA, where the required dynamic range will be an order of magnitude beyond any existing array. Because redundancy gives a better handle on characterizing the state of the system, it provides in addition a model-independent diagnostic tool for subsystems such as a station.

Acknowledgements. The authors would like to heartfully thank Mariana Ivashina and Rob Maaskant for their generous and helpful discussions about antenna and system issues, Rob Maaskant and Michel Arts for their help with CAESAR simulations, and Sarod Yatawatta, Jan Noordam, Wim Brouw, Jaap Bregman, and Ger de Bruyn for their very useful advice and discussions. They also thank the anonymous referee, whose useful comments have considerably improved the clarity of this paper.

\section{References}

Ardenne, A., Wilkinson, P. N., Patel, P. D., \& Vaate, J. G. B. 2004, Exp. Astron., 17,65

de Vos, M., Gunst, A. W., \& Nijboer, R. 2009, IEEE Proc., 97, 1431

Ivashina, M. V., Iupikov, O., Maaskant, R., van Cappellen, W. A., \& Oosterloo, T. 2011, IEEE Trans. Antennas Propag., 59

Jeffs, B. D., Warnick, K. F., Landon, J., et al. 2008, IEEE J. Selected Topics in Signal Processing, 2, 635

Kant, G. W., Patel, P. D., Wijnholds, S. J., Ruiter, M., \& van der Wal, E. 2011, IEEE Trans. Antennas Propag., 59, 1990

Liu, A., Tegmark, M., Morrison, S., Lutomirski, A., \& Zaldarriaga, M. 2010, MNRAS, 408, 1029

Maaskant, R. 2010, Ph.D. Thesis, Technische Universiteit Eindhoven

Maaskant, R., \& Yang, B. 2006, in The European Conference on Antennas and Propagation: EuCAP 2006, ESA SP, 626

Maaskant, R., Tijhuis, A. G., Mittra, R., et al. 2008, Proc. 38th European Microwave Conference, Amsterdam, 837

Noordam, J. E., \& de Bruyn, A. G. 1982, Nature, 299, 597

Prakash, V., \& Mittra, R. 2003, Micr. Opt. Technol., 36, 95

Svantesson, T. 1998, in Statistical Signal and Array Processing, Proc. Ninth IEEE SP Workshop, 232

van Ardenne, A., Bregman, J. D., van Cappellen, W. A., Kant, G. W., \& de Vaate, J. G. B. 2009, IEEE Proc., 97, 1531

Wieringa, M. 1991, Ph.D. Thesis, Rijksuniversiteit te Leiden

Wijnholds, S. J., \& van der Veen, A.-J. 2009a, IEEE Transactions on Signal Processing, 57, 3512

Wijnholds, S. J., \& van der Veen, A.-J. 2009b, 17th European Signal Processing Conference, Glasgow, UK, 24

Wijnholds, S. J., \& van Cappellen, W. A. 2011, IEEE Trans. Antennas Propag., 59,1981

Wijnholds, S. J., van der Tol, S., Nijboer, R., \& van der Veen, A.-J. 2010, IEEE Signal Processing Magazine, 27, 30

Yeo, J., Prakash, V., \& Mittra, R. 2003, Micr. Opt. Technol, 39, 456 


\section{Appendix A: More plots of residuals for corrected redundant visibilities}

We present additional plots of the residuals for the corrected redundant visibilities of our observational campaign on 24 November 2009. Figure A.1 shows similar results of the same data set (captured at 21:29:04 UTC, when CasA was at high elevation) presented in Fig. 8 but for different baselines, shown in the rightmost panel.
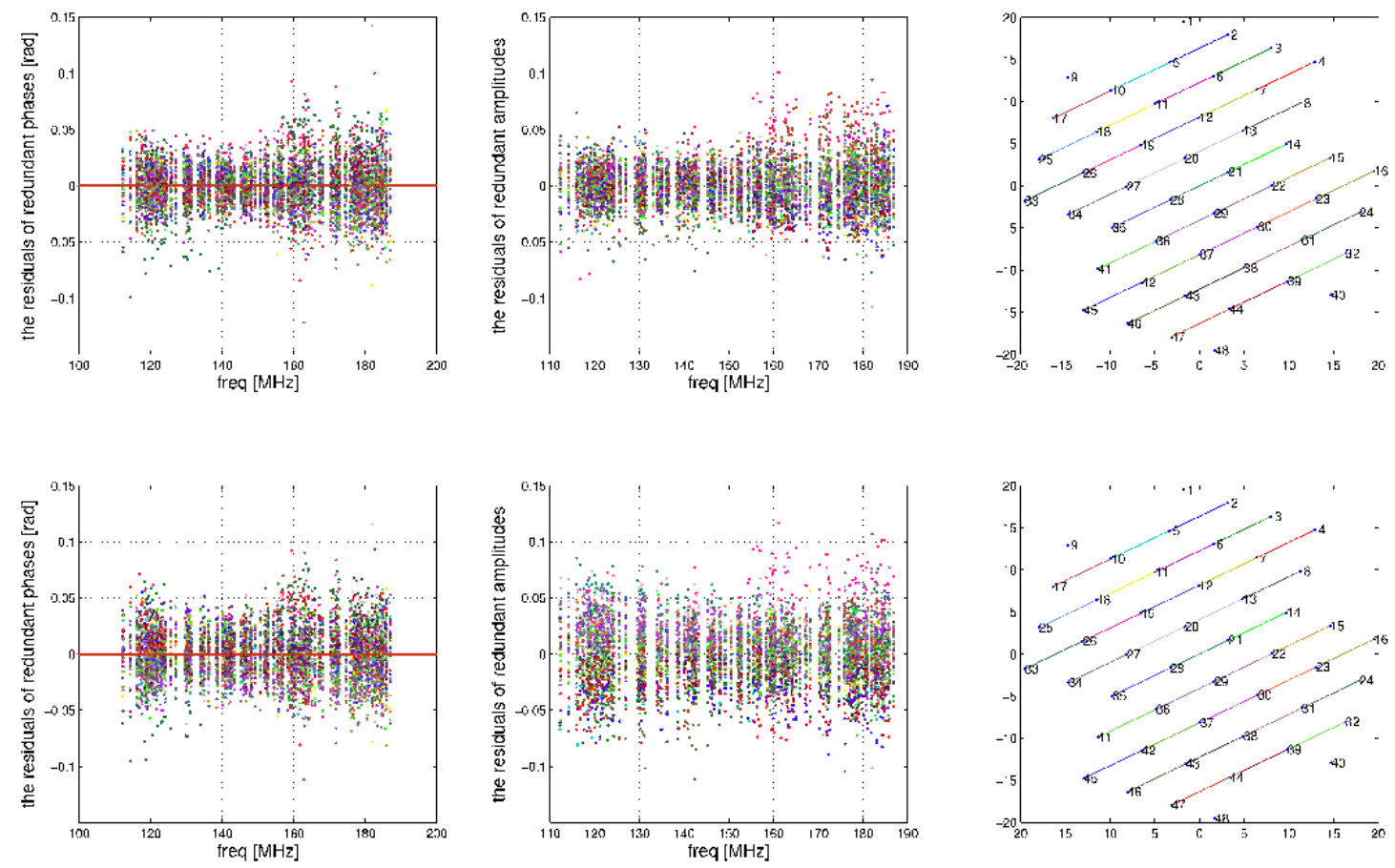

Fig. A.1. Plots of the residuals for corrected redundant visibilities in terms of phases and amplitudes on a given set of redundant baselines. The first row shows the results after redundancy calibration. The second row shows the result afters model-based calibration. The data is taken from an observation done on 24 November 2009 at 21:29:04 UTC, when CasA was at high elevation. The station configuration of RS208 is shown in the rightmost panel. The corresponding redundant baselines to each redundant visibility are also depicted on the station layout, with the same color code.
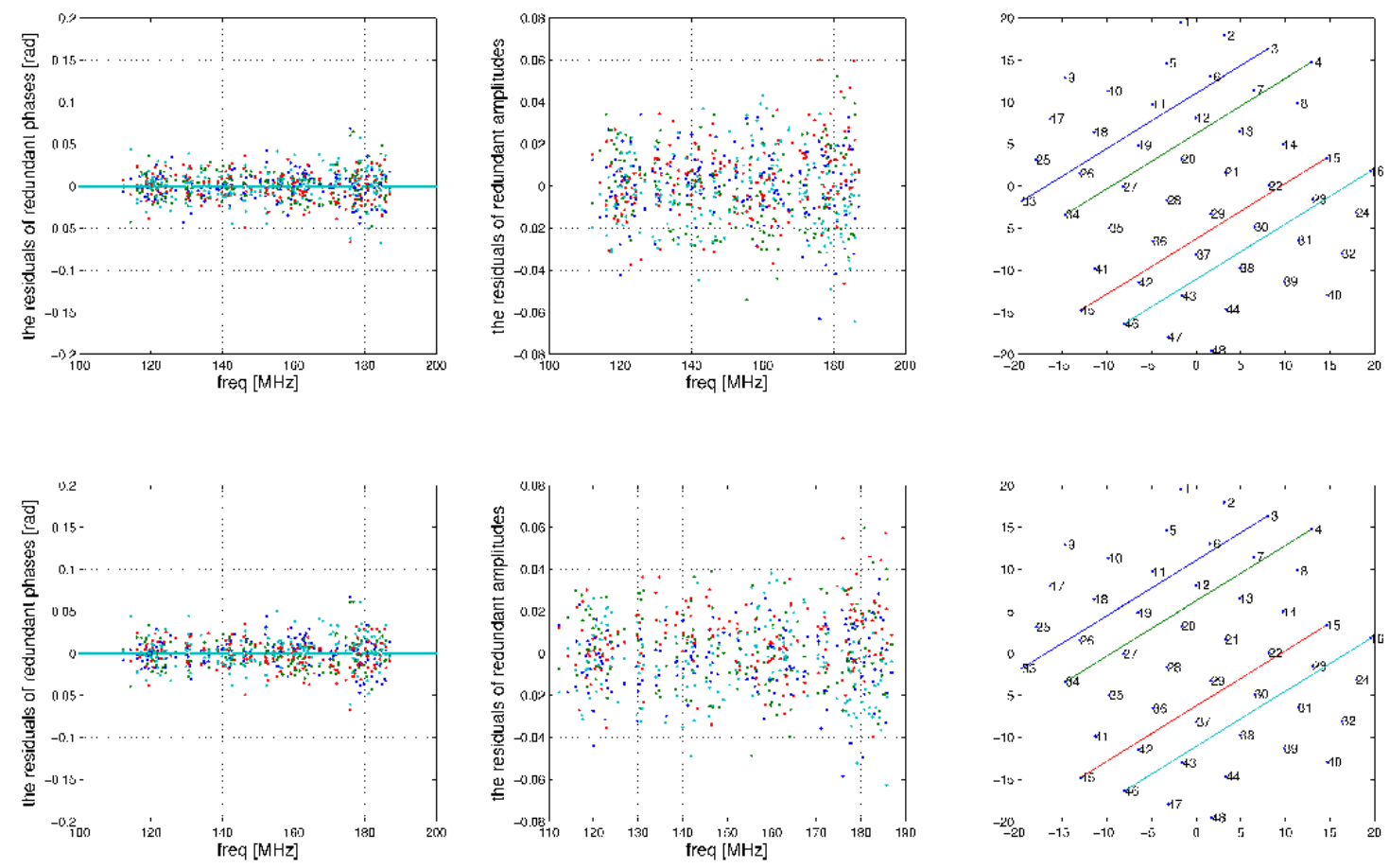

Fig. A.1. continued. 
A\&A 545, A108 (2012)
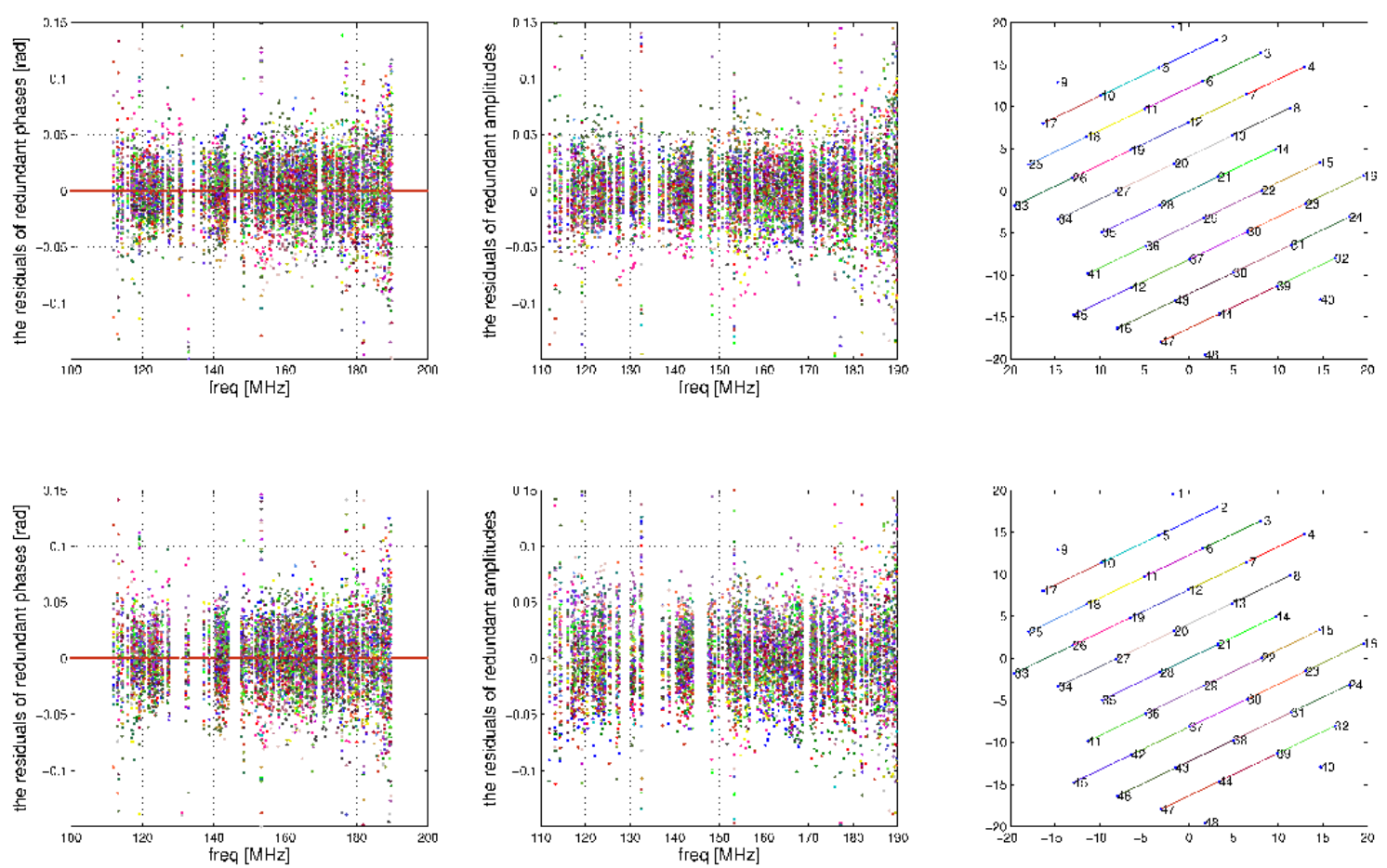

Fig. A.2. Plots of the residuals for corrected redundant visibilities in terms of phases and amplitudes on a given set of redundant baselines. The first row shows the results after redundancy calibration. The second row shows the result afters model-based calibration. The data is taken from the observation done on 24 November 2009 at 15:25:43 UTC, when CasA was at low elevation. The station configuration of RS208 is shown in the rightmost panel. The corresponding redundant baselines to each redundant visibility are also depicted on the station layout, with the same color code.
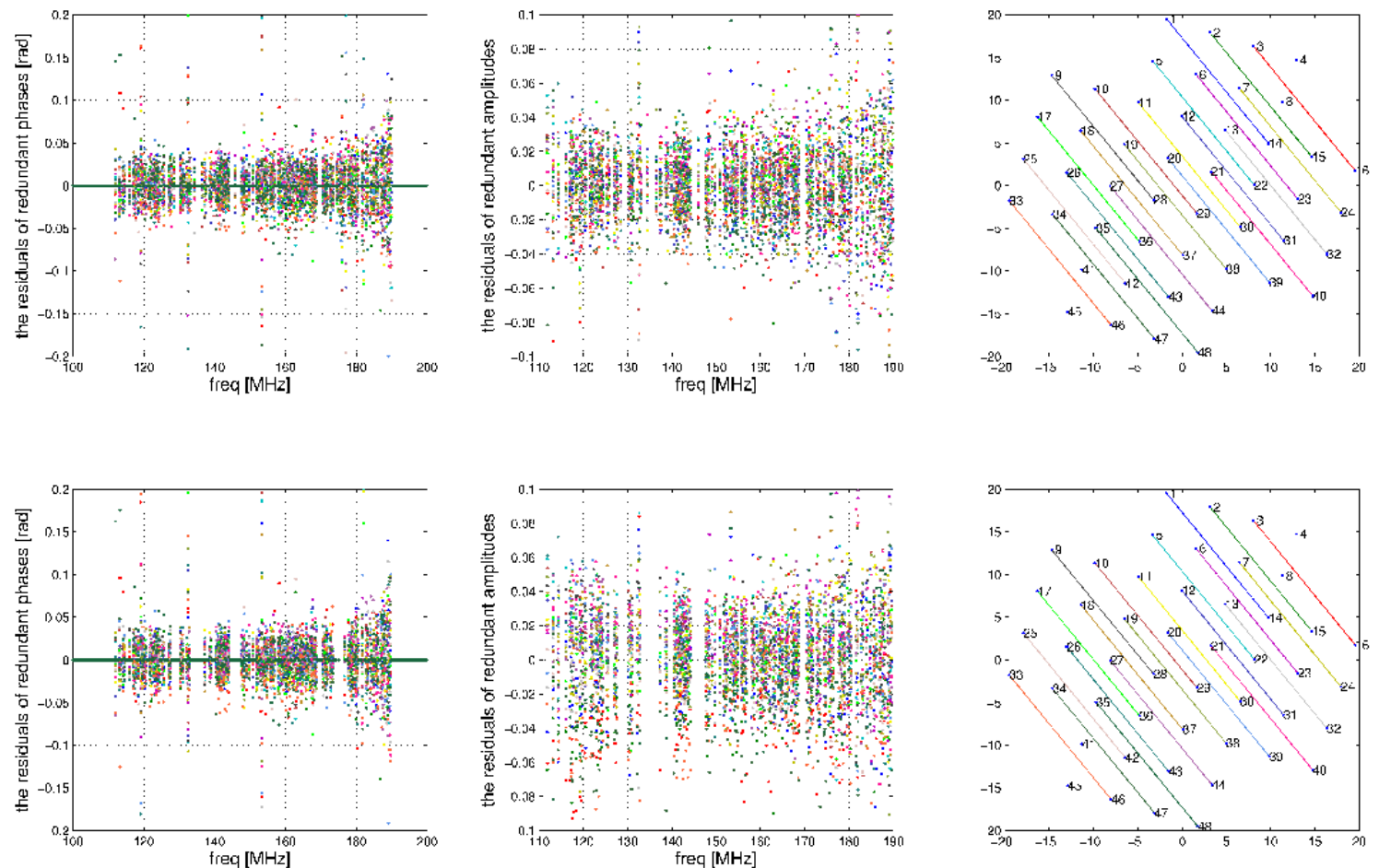

Fig. A.2. continued. 
P. Noorishad et al.: Redundancy calibration of phased-array stations
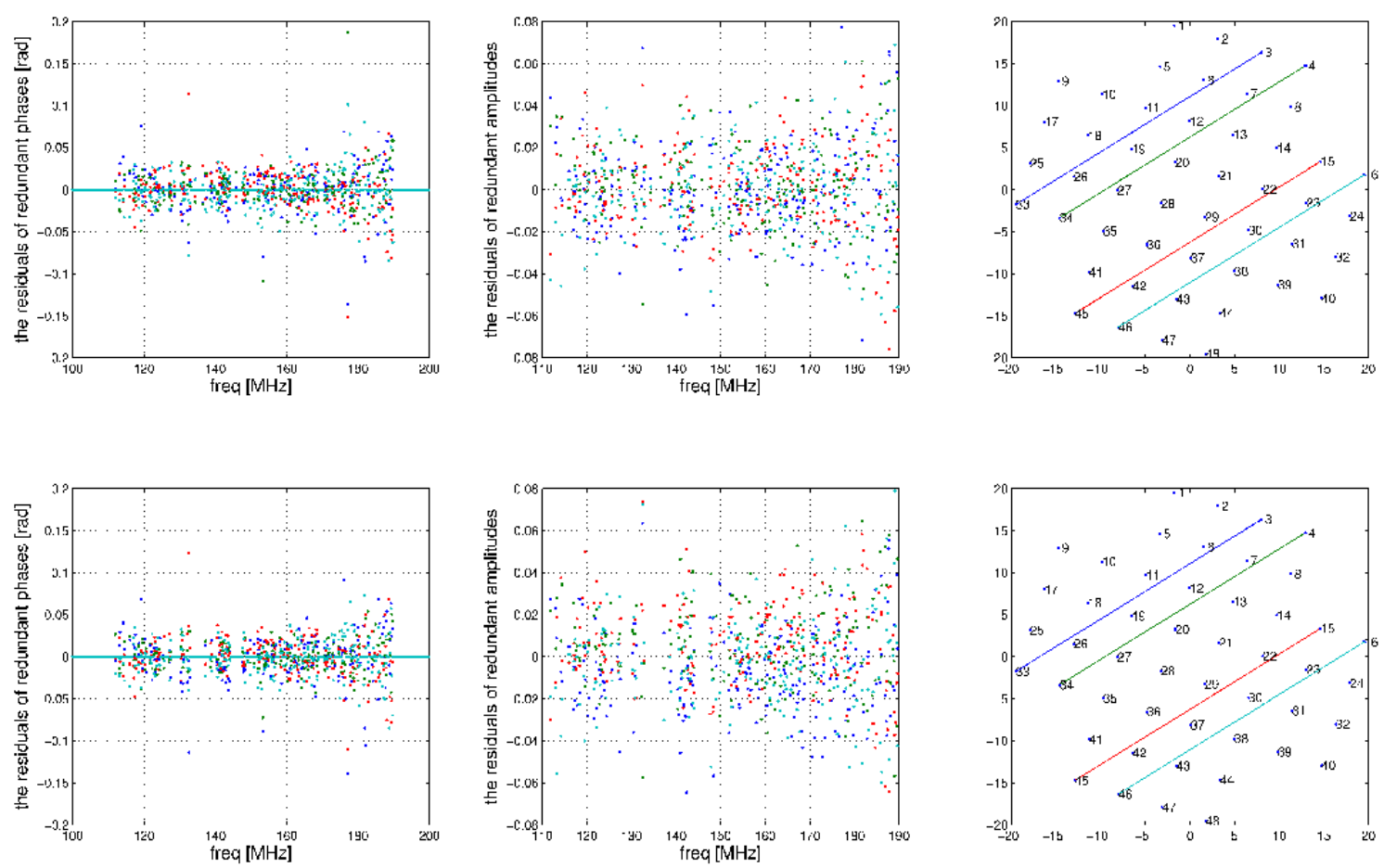

Fig. A.2. continued. 